\title{
A catalog of observed nuclear magnitudes of Jupiter family comets $^{\star, \star \star}$
}

\author{
G. Tancredi ${ }^{1}$, J.A. Fernández ${ }^{1}$, H. Rickman ${ }^{2}$, and J. Licandro ${ }^{3}$ \\ 1 Departamento de Astronomía, Facultad de Ciencias, Iguá 4225, 11400 Montevideo, Uruguay \\ 2 Astronomiska Observatoriet, Box 515, S-75120 Uppsala, Sweden \\ 3 Instituto de Astrofísica de Canarias, E-38200 La Laguna, Tenerife, Spain
}

Received January 7; accepted July 5, 2000

\begin{abstract}
A catalog of a sample of 105 Jupiter family (JF) comets (defined as those with Tisserand constants $T>2$ and orbital periods $P<20 \mathrm{yr}$ ) is presented with our "best estimates" of their absolute nuclear magnitudes $H_{\mathrm{N}}=$ $V(1,0,0)$. The catalog includes all the nuclear magnitudes reported after 1950 until August 1998 that appear in the International Comet Quarterly Archive of Cometary Photometric Data, the Minor Planet Center (MPC) data base, IAU Circulars, International Comet Quarterly, and a few papers devoted to some particular comets, together with our own observations. Photometric data previous to 1990 have mainly been taken from the Comet Light Curve Catalogue (CLICC) compiled by Kamél (1991). We discuss the reliability of the reported nuclear magnitudes in relation to the inherent sources of errors and uncertainties, in particular the coma contamination often present even at large heliocentric distances. A large fraction of the JF comets of our sample indeed shows various degrees of activity at large heliocentric distances, which is correlated with recent downward jumps in their perihelion distances. The reliability of coma subtraction methods to compute the nuclear magnitude is also discussed. Most absolute nuclear magnitudes are found in the range $15-18$, with no magnitudes fainter than $H_{\mathrm{N}} \sim 19.5$.

The catalog can be found at: http://www.fisica.edu.uy/ $\sim$ gonzalo/catalog/.
\end{abstract}

Key words: Comets: general — catalogs - techniques: photometric

Send offprint requests to: G. Tancredi, e-mail: gonzalo@fisica.edu.uy

* Table 2 and Appendix B are only available in electronic form at http://www.edpsciences.org

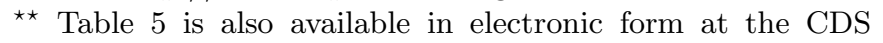
via anonymous ftp to cdsarc.u-strasbg.fr (130.79.128.5) or via http://cdsweb.u-strasbg.fr/Abstract.html

\section{Introduction}

The determination of comet brightness has been one of the important goals of comet observers for the last century or so. The challenge of making reliable estimates has been enormous since one is dealing with extended sources in motion with respect to background stars. Most estimates of comet magnitudes have been done by visual or photographic methods. However, CCD observations have become increasingly common in recent years.

Most of the determined comet magnitudes have referred classically to the magnitude of the gaseous coma surrounding the comet nucleus. These have been known as "total" magnitudes. Max Beyer was one of the pioneers in trying to determine visually magnitudes of the comet nucleus during the 1930's and -40 's. His nuclear magnitudes were grossly underestimated (i.e. the nucleus brightness overestimated) due to coma contamination. The following most serious attempt at determining a homogeneous data set of nuclear magnitudes was carried out by Elizabeth Roemer during a time span of more than 25 years (from 1950 to the late seventies). Roemer used photographic plates taken with the, at the time, Lunar and Planetary Laboratory 1.4-m at the Catalina Station and the Steward Observatory 2.3-m at Kitt Peak (see, for instance, Roemer 1976).

As Roemer was carrying out her photometric work, progress was made in another observational front: spacebased ultraviolet observations allowed the observation of the Lyman-alpha line of hydrogen at $1216 \AA$ and thereby an estimate of the water production rate. Other species were also observed in the UV, and a very important data base for inferring $\mathrm{H}_{2} \mathrm{O}$ production rates came from IUE observations of the $\mathrm{OH}$ bands near $309 \mathrm{~nm}$. In parallel, radio observations of the $18-\mathrm{cm}$ lines of $\mathrm{OH}$ allowed an independent determination of the water production rate. A'Hearn \& Millis (1980) introduced narrow band filters to isolate different molecular species (e.g. $\mathrm{C}_{2}, \mathrm{C}_{3}, \mathrm{CN}, \mathrm{OH}$ ) 
in their ground-based photometric observations of comets with the aim of determining their production rates. The study of gas production rates of different species thus gave a confirmation that $\mathrm{H}_{2} \mathrm{O}$ controls the cometary outgassing near the Sun and should be the most abundant volatile in comets.

In the presence of data on $\mathrm{H}_{2} \mathrm{O}$ production rates, cometary nuclear magnitudes took on a new meaning, viz., in terms of the sizes and activity levels of the nuclei. However, the determination of the nuclear size is not straightforward, since it also depends on the albedo of the nuclear surface. Delsemme \& Rud (1973) attempted for the first time to derive both the nuclear radii and albedos of comets C/1969 T1 (Tago-Sato-Kosaka), C/1969 Y1 (Bennett) and 2P/Encke (1971 perihelion passage) by combining their measured water production rates close to perihelia with Roemer's nuclear magnitudes obtained at large heliocentric distances. Even though the derived albedos for C/1969 T1 Tago-Sato-Kosaka and C/1969 Y1 Bennett were too high for what one would expect of dirty ice surfaces, theirs was nevertheless a pioneering and influential approach to this problem.

Meanwhile, near the end of the "Roemer era" there was still serious doubts that a comet nucleus would have been resolved in any case (e.g. Sekanina 1976), so most researchers tended to regard "nuclear" magnitudes as the magnitude of the solid nucleus plus an inner coma. During the 1980 's, comet $1 \mathrm{P} /$ Halley of course became the main target of cometary research and the great opportunity to observe for the first time a bare nucleus by means of spacecraft fly-by. This goal was successfully accomplished by the Giotto and Vega missions. The 1P/Halley nucleus turned out to be an elongated body of $14.2 \mathrm{~km} \times 8.2 \mathrm{~km} \times 7.5 \mathrm{~km}$ of very low albedo and with an active surface area no greater than about $15 \%$ of the total (Keller et al. 1987). The knowledge of the nucleus size allowed for the first time a direct comparison between comet size and the earlier estimates of the nuclear magnitude based on ground-based CCD observations of $1 \mathrm{P} /$ Halley at distances greater than 8 AU. The nucleus size derived by Jewitt \& Danielson (1984) from these distant observations turned out to be a factor of two smaller than the size derived by the space missions. In any case, the new technology of CCD cameras attached to large telescopes proved to be very promising at observing distant comets - where they are presumably little active or inactive - with the scope of deriving nuclear magnitudes and sizes.

CCD photometry of comets became of widespread use in the post-Halley era, with observers like David Jewitt, Karen Meech, Tom Gehrels and James Scotti among the pioneers in the use of this new technology for the study of comets. The much higher sensitivity of CCD detectors and the use of medium-sized to large telescopes allowed the observation of a large number of comets beyond $3 \mathrm{AU}$ and the early recovery of short-period comets when they had little activity or no activity at all.
Of particular relevance is the work of James Scotti with the 91-cm Spacewatch telescope. Scotti has not only observed distant comets systematically, contributing to the early recovery of a large fraction of the short-period comets, but due to the high-resolution surface photometry capacity of its CCD exposures, he has also introduced a method of coma subtraction to derive an improved magnitude of the nucleus (see Sect. 3.2).

The latest improvement on the quality of the observations has been achieved with the data taken by the Hubble Space Telescope, obtained under the leadership of Philippe Lamy. The outstanding image quality obtained by the HST allows the application of a more refined coma subtraction technique even in highly active comets (Lamy \& Toth 1995 and later references, see below).

In summary, the last decade of the century has seen a growing activity in photometric observations of distant comets. However, overall only a wealth of photometric data has been produced without detailed analysis of its physical meaning. There are just a few exceptions for some particular comets. We now deem that the time is ripe to undertake a broad analysis of the observed nuclear magnitudes. We restrict our sample to the comets of the Jupiter family (JF) that we define following Valsecchi (1992) as those with Tisserand constants $T>2$ and periods $P<$ $20 \mathrm{yr}$ (there are so far only four comets with $P<$ 20 yr that have $T<2$ ). We choose this population for two reasons: (1) a large fraction of the JF population has been extensively observed photometrically, whereas long-period comets and Halley-type comets show only scattered data; and (2) we would like to analyze a homogeneous population, presumably coming from the same source region (in this case the Edgeworth-Kuiper belt). Admittedly, we do not know to what extent this presumption is correct, since the JF population may be contaminated with comets coming from other sources as, for instance, the Oort cloud (Bailey 1986) or the Trojans (Rabe 1972), it is likely that such contamination represent only a minority of the whole population.

The present catalog is a continuation of a project started several years ago (see Fernández et al. 1992), that includes our own observational program (Licandro et al. 1999a). A theoretical analysis based on the information described here is presented separately (Fernández et al. 1999).

\section{What is a nuclear magnitude?}

A simple answer to this question could be: It is the magnitude of a comet nucleus. But then we are faced with two obvious difficulties: How do we estimate a nuclear magnitude?; and can the comet nucleus actually be observed? For practical purposes, the nuclear magnitude may be defined as the rather sharp condensation of light in the inner coma, and this concept has been adopted by many 


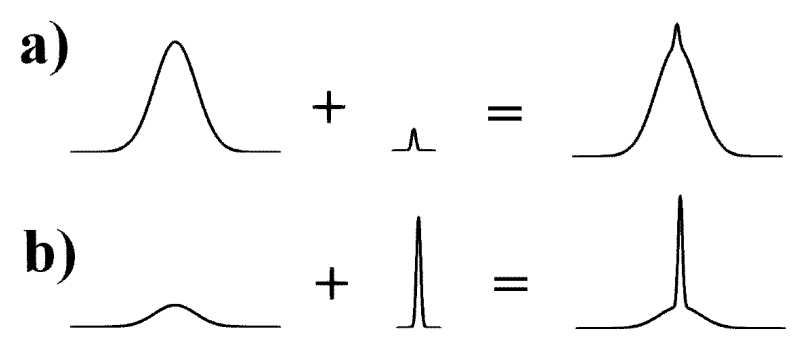

Fig. 1. Schematic representation of the coma and nucleus profiles separately (left-hand side); and the addition of the two profiles (right-hand side) for a: a) coma-dominated profile; and b) nucleus-dominated profile

observers, mainly amateur astronomers. They estimate the nuclear magnitude by measuring the total flux inside a small disc centered on the brightest peak. Unfortunately, such measurements have little physical meaning. If one wants to get some hint on the actual nucleus size, one should strive, as far as possible, to measuring solely the light reflected by the solid nucleus. In such an ideal case the brightness would indeed be proportional to the observed geometric cross-section of the nucleus. Given the smallness of the nucleus and the distance at which it is generally observed, it would necessarily appear as a starlike source. Thus a true and straightforward nuclear magnitude can only be estimated if the comet has a stellar appearance; i.e., no trace of coma should be detected.

In other words, the brightness of an active comet is the sum of the coma and the nucleus brightness. This total, i.e., coma plus nucleus, intensity on the focal plane can be represented as a $3-\mathrm{D}$ surface. Assuming an axisymmetric surface brightness of the coma and a Gaussian-like Point Spread Function (PSF), each brightness profile would have a bell shape as shown in Fig. 1. Unless the nuclear brightness is much higher than that of the coma, the flux measured in a small disc centered on the brightest peak would have a non-negligible contribution from the coma. Thus, such a nuclear magnitude would not have a straightforward physical interpretation but only give an ill-defined upper limit to the true nuclear brightness. Accordingly, we adopt the definition that: A nuclear magnitude corresponds to the total flux coming from the solid nucleus of the comet. We should, however, bear in mind that its determination is a very difficult task.

The photometric cross-section $S$ of a nucleus of radius $R_{\mathrm{N}}$ is given by

$\log \left(p_{\mathrm{v}} S\right)=16.85+0.4 \times\left[m_{\odot}-H_{\mathrm{N}}\right]$

where $p_{\mathrm{v}}$ is the geometric albedo in the visual, $S=\pi R_{\mathrm{N}}^{2}$ is expressed in $\mathrm{km}^{2}, H_{\mathrm{N}} \equiv V(1,1,0)$ is the absolute (visual) nuclear magnitude of the comet (the apparent magnitude at $1 \mathrm{AU}$ from the Sun and the Earth and zero phase angle) and $m_{\odot}=-26.77$ the apparent (visual) magnitude of the
Sun. Almost all periodic comets so far studied have very low geometric albedos $\left(p_{\mathrm{v}} \approx 0.02-0.05\right)$ (Hartmann et al. 1987; Jewitt 1996; Weissman et al. 1989).

\section{The data}

\subsection{The sources}

About half of the magnitudes included in this catalog have been taken from the compilation of cometary magnitudes collected by Kamél (1991) (the "Comet Light Curve Catalogue" - CLICC). Kamél mainly used two sources for references: Astronomischer Jahresbericht for the period 1899-1968 and Astronomy and Astrophysics Abstracts for the period 1969-1989. A large part of his data set comes from Green's (1988) Archive of Cometary Photometric Data. The ending date for CLICC was December 31, 1989. Although for sure it is not complete, CLICC is the largest data set for cometary magnitudes available for that period of time.

We have added magnitude measurements for the period January 1, 1990 to August 1998 taken from the Minor Planet Center (MPC) data base. We downloaded the observations for all the JF comets from the Extended Computer Service of the MPC. It should be borne in mind that the MPC data base is mainly for astrometric purposes, and the reported magnitudes are not of homogeneous quality. There is a distinction between total and nuclear magnitudes, but no information is given on how the nuclear magnitudes were estimated. Furthermore, the passband of the measurement is not mentioned, and the only information about the observer and his/her instrument is the observatory code.

We have added a few extra observations that were not included in the MPC data base but appeared in the IAU Circulars or International Comet Quarterly. Data from a few papers devoted to particular comets, published after 1990, have also been included (Mueller 1992; Meech et al. 1993; Chen \& Jewitt 1994; Lamy \& Toth 1995; Mueller \& Ferrin 1996; Lamy et al. 1996; Lamy et al. 1998a; Lamy et al. 1998b) as well as our own set of nuclear magnitudes (Licandro et al. 1999a).

From these data sources, we have taken all the nuclear magnitudes and total magnitudes observed at heliocentric distances $r>3$ AU observed after 1950. The cometary activity, on average, is a strong function of the heliocentric distance. The inner coma brightness in the visual is largely due to the presence of a dust cloud surrounding the nucleus. The sublimation of volatile species (mainly $\mathrm{H}_{2} \mathrm{O}$ ) drives the ejection of dust from the nucleus. At $r>3 \mathrm{AU}$, cometary activity is expected to be very low because the $\mathrm{H}_{2} \mathrm{O}$ sublimation rate is tiny due to the low surface temperature. Total magnitudes at those heliocentric distances might thus be close estimates of the true nuclear magnitudes. Yet, this cannot be taken 
as an absolute rule: some comets keep very active at heliocentric distances well above 3 AU, presumably due to outgassing of species more volatile than $\mathrm{H}_{2} \mathrm{O}$ (e.g., $\mathrm{CO}$ and/or $\mathrm{CO}_{2}$ ). Therefore, we cannot take for granted that magnitudes measured at $r>3$ AU never have strong coma contamination.

We find that $59 \%$ of the data points are magnitudes reported as nuclear, though there are different criteria among the observers to define a nuclear magnitude (see Sect. 3.2). The number of pre-perihelion observations is similar to that of post-perihelion observations.

From thermal lag considerations, one might expect some asymmetry between the outbound and inbound branches of the comet's orbit, such that after passing aphelion, the JF comets might be at a lower level of outgassing activity (i.e., closer to showing the bare nucleus). Yet, the photometric data is still too sparse to confirm this theoretical presumption. A better photometric coverage of JF comets along their orbits is necessary before reaching any conclusion regarding the part of the orbit at which JF comets reach their lowest level of activity (or complete inactivity).

Some considerations are presented in Sect. 3.3 to justify the deletion of some data. Table 1 presents a summary of the sources and a general classification of the data. The numbers listed correspond to the data remaining after the deletions mentioned in Sect. 3.3.

Another piece of very valuable information would be the negative observations, i.e., in case the comet was not found, an estimation of the limiting magnitude on the skyfield where one would expect to find the comet. This would give a lower limit to the brightness that in many cases could be lower than a positive detection. There are two reasons why we do not intend to collect these data: i) most of the authors, do not publish the negative observations, e.g. though we have a large data set of negative detections from our observing runs, we do not include them in our paper (Licandro et al. 1999a); ii) the negative observations could be due to a cometary magnitude fainter than our detection limit, but also could be due to ephemeris uncertainties. Remember that comets are subjected to nongravitational forces that are, generally, poorly known; they could produce differences between the real and expected position larger than several arc-minutes. With CCD fields of a few arc-minutes, the comet could easily be out of the field or far from the center. Our own experience tells us that it is very hard to find a moving object close to the detection limit that it is not close to the field center. Many negative reports could reflect uncertanties in the computed orbit rather than a faint magnitude.

\subsection{Main categories of observers}

There are four data sets that deserve a detailed analysis: long-focus photographic observations by Elizabeth
Table 1. Statistics of our data sets

\begin{tabular}{|c|c|}
\hline Number of observations ${ }^{(1)}$ & 3990 \\
\hline Data taken from CLICC & 1474 \\
\hline Data taken from MPC & 2392 \\
\hline Data taken from other sources ${ }^{(2)}$ & 124 \\
\hline Observations pre-perihelion & 1968 \\
\hline Observations post-perihelion & 2022 \\
\hline Reported nuclear magnitudes ${ }^{(3)}$ & 1952 \\
\hline Total magnitudes at $r>3 \mathrm{AU}^{(3)}$ & 1073 \\
\hline Scotti's total magnitudes at $r \leq 3 \mathrm{AU}^{(4)}$ & 514 \\
\hline Scotti's total magnitudes at $r>3 \mathrm{AU}^{(4)}$ & 553 \\
\hline Scotti's nuclear magnitudes ${ }^{(5)}$ & 412 \\
\hline Roemer's magnitudes & 700 \\
\hline CCD nuclear magnitudes ${ }^{(6)}$ & 312 \\
\hline
\end{tabular}

(1) For consistency with other observers, the observations by J. Scotti reported as total but taken at $r \leq 3 \mathrm{AU}$ are not included in these numbers.

(2) Observations taken from the ICQ after 1990 that did not appear in the MPCs (25), personal communication by C. Hergenrother (6), our own observations (48), data taken from the literature (45).

(3) Observations by J. Scotti are not included.

(4) Magnitudes reported as total.

(5) Magnitudes reported as nuclear, in most cases Scotti has applied a coma reduction method.

(6) Only the observations with medium and large aperture telescopes are included. This number may be underestimated, because for many observers we do not know which telescope was used.

Roemer; James Scotti's Spacewatch observations; recent data taken by other professional astronomers using CCD cameras attached to medium- and large-size telescopes; and, finally, amateur observations with small-size telescopes. There is finally a fifth data set that is rapidly increasing in importance: we refer to the recently published observations of cometary nuclei using the Hubble Space Telescope.

Elizabeth Roemer started her observations in 1950. Almost every comet to appear in the following 25 years was photographed by her. She used a $1.4-\mathrm{m}$ and a $2.3-\mathrm{m}$ telescopes at Catalina Station and Kitt Peak, respectively. The trend at that time was to estimate the nuclear magnitude by visual inspection of the plates, where the sharp concentration of the inner coma was actually measured. Roemer argued that long-focus instruments and photographic techniques were best suited for the observation of quasi-stellar comets, where little or no traces of the coma were recorded. The plates, originally taken for astrometric purposes, were used for photometric estimates of the nuclear condensations. The photometry (often around 
$20^{\text {th }}$ magnitude) could not be done accurately with the aid of nearby photoelectric sequences. The apparent photographic magnitudes observed were hence reported only in terms of whole or half magnitude units.

Contributing $18 \%$ of the entries in our data base, Roemer's material is obviously of great interest. During the Roemer era, her observations were generally the faintest, indicating that she indeed came closest to measuring the actual nuclei, whereas other observers were more influenced by coma contamination. However, when viewed from a present-day perspective, it is clear that there is no guarantee that her stellar sources would represent the bare nuclei, and generally speaking, CCD frames offer much better opportunities. This is especially true in the many cases when they have reached $2-3$ magnitudes deeper than Roemer's plates and caught the comets closer to their aphelia.

The second large data set from a single observer corresponds to James Scotti's observations with the Spacewatch telescope. This is a 91-cm Schmidt telescope at Kitt Peak dedicated to the discovery and follow-up of Near-Earth Objects. Scotti started his cometary observations with T. Gehrels, but since the late 80's he has conducted his own program for cometary photometry. $24 \%$ of the entries in our data base corresponds to his observations (we do not take into account the reports of total magnitudes at $r<3 \mathrm{AU}$ ). During the 90's he has recovered many comets at large heliocentric distances (in the sense of the first observation after aphelion passage).

Scotti has developed a tentative method to face the problem of nuclear magnitude determination in active comets. Though a detailed description of the method has yet to be published, we asked Scotti for a brief sketch of it. As described in Sect. 1, the brightness profile of an active comet is the addition of the brightness profile of the coma plus a stellar-like nucleus. Assuming an optically thin coma, the typical width of the nucleus contribution is on the order of two times the Full Width at Half Maximum of the PSF (the seeing). At a distance a few times the radius of the seeing disk from the photometric centre of the comet, the contribution to the profile mainly comes from the coma. Scotti then takes a thin annulus of this radius and computes the mean surface brightness $(\sigma)$. He assumes a constant coma surface brightness inward of that annulus. From the total flux of a disk centered on the brightest pixel he subtracts a coma flux corresponding to: $\sigma \times A$ ( $A$ - the disk area). The remaining flux supposedly corresponds to the contribution of the nucleus. A nuclear magnitude can thus be computed.

Since $10 \%$ of our data correspond to coma-corrected observations thus derived by Scotti, let us scrutinize these a little closer. Several drawbacks may question the validity of this coma-correction method:

i) The assumption of an optically thin coma, in particular in the region very close to the nucleus. A few observed near stellar occultations suggested that extinctions ranging from a few percent to a few tens of percent of the starlight were present in stars passing at distances of a few hundred $\mathrm{km}$ from the comet nucleus (Larson \& A'Hearn 1984; Eritsyan \& Akhverdyan 1987; Ninkov 1994; Fernández et al. 1999). However, other occultations of stars by JF comets have not shown any appreciable extinction. Licandro et al. (1999b) have studied several occultations of stars by the inner coma of comets $81 \mathrm{P} / \mathrm{Wild}$ 2, 69P/Taylor, and 78P/Gehrels 2. They concluded that no extinction is detected even for stars that passed very close to the nucleus (a few hundred $\mathrm{km}$ ). They also discussed methodological problems involved in such studies, which may produce spurious drops of the star brightness. In any case the assumption of an optically thin coma, supported by model predictions (see Appendix A), seems to have observational support. Thus, we do not expect any significant trend for overcorrections due to coma extinction.

ii) The assumption of a constant coma surface brightness $(\sigma)$ inside the annulus. It is observed that $\sigma$ is a function of $1 / \rho$ ( $\rho$ is the angular distance from the center) for intermediate distances to the nucleus, but it should level off as $\rho \rightarrow 0$. However, a monotonic increase is expected as $\rho \rightarrow 0$. Since Scotti assumes a constant coma surface brightness inside the annulus, he tends to underestimate the true coma contribution inside the small disc. The amount of the underestimation would depend on the size of the coma and the radius of the annulus. Grosser underestimations could be expected for small apparent comae, typical of JF comets with large perihelion distances, since the annulus would fall near the coma's edge.

iii) The background "sky". There may also be problems with unresolved background objects contaminating the "sky" level underlying the coma. Sometimes the annulus may contain a significant extra brightness from such objects, and then the coma contribution may be overestimated and the nuclear magnitude obtained after the coma substraction may be fainter than the real one. However, it may also happen that the background is more contaminated in the interior of the annulus than within it, and thus the expectation is relatively few cases of gross overcorrections compensated by a larger number of slighter undercorrections.

iv) The difference between the total and coma fluxes inside the small disc may be close to the noise level. Let us consider a difference of 4 magnitudes between the total and corrected nuclear magnitudes. If the surface brightness varies as $\sigma \propto 1 / \rho$, the coma flux inside a disc of radius $\rho$ is directly proportional to $\rho$. Assuming a coma diameter of $1^{\prime}$, the coma brightness inside a central disc of $10^{\prime \prime}$ diameter would be seven times the nuclear brightness. The actual photometric measurement would be given by the coma and the nucleus fluxes plus the sky background and the read-out. By Poisson statistics, the noise in the measurement would be on the order of the square root 
of the value. Applying Scotti's method, the nuclear flux would be computed from the difference of two similar quantities that are many times larger than this difference, and the statistical uncertainties of each would be on the order of the square root of the value. We may expect a significant random scatter in such situations.

v) The color of the magnitude. Scotti's CCD frames are taken without filters. He uses solar analogs as standards to estimate his magnitudes, so these resemble the standard visual magnitudes, though some small color corrections may be involved to define them in the Bessel or Kron-Cousins systems. Conceivably, a systematic error of $\sim 0.1-0.2$ magnitudes may be involved, but this is not very important compared with other sources of errors. A larger non-systematic error (may be even more than $1 \mathrm{mag}$ ) could be foreseen if the observer does not calibrate the observations on a nightly basis, a fact we do not know.

From the previous discussion we can conclude that Scotti's coma subtraction method may be rather uncertain, at least in the cases of very active comets, as shown by the wide spread of the estimated nuclear magnitudes in some cases; for low-active comets it may give more useful estimates of the nuclear magnitudes (see Sect. 8.2 for a more detailed analysis).

The majority of Scotti's reports are taken from the MPCs. We have already mentioned the drawbacks of these data for photometric purposes. It is only mentioned that the observed magnitudes were total or nuclear, but nothing is said e.g. if a total magnitude corresponds to a comet with stellar appearance (in which case it can be considered as a typical nuclear magnitude), or if the coma subtraction method was applied to the reported nuclear magnitude. In many cases, for similar observing days, Scotti reported total as well as nuclear estimates. We decided to include all his nuclear as well as total reports (even those at $r<$ $3 \mathrm{AU}$ ); in that way we were able to analyse the relative contribution of the nucleus and the coma to the total magnitude and the validity of the coma subtraction method. Note that almost half of the observations in our data base correspond to Roemer or Scotti.

Professional astronomers using medium and large telescopes (say, larger than $1.5 \mathrm{~m}$ aperture) with CCDs constitute another important group of observers to be discussed. The pioneer in this field was David Jewitt, who started in 1984 a photometric program of distant comets, first with Karen Meech and later with Jane Luu. During the 90's several other groups have engaged in the difficult task of detecting comets close to their aphelia; e.g., Larson \& Hergenrother, Meech et al., Mueller et al. and our group (Licandro et al. 1999a). These groups use high quality CCDs attached to large telescopes in sites with good seeing conditions; the detection of even a weak coma is much easier and the distinction between nuclear and total magnitudes more clear. The members of these groups define the nuclear magnitude as the total magnitude of the comet when it has a stellar appearance. If there is no coma, this magnitude would correspond to the definition of nuclear magnitude we have adopted in Sect. 2 .

Finally, we have the contribution of many amateur astronomers. The amateur data is very inhomogenous. Some consider a magnitude as nuclear only if they see the comet inactive (no detectable coma), but most of them define the nuclear magnitude as the magnitude of the central brightness. In the past, they may even have tried some methods for "better" estimates of nuclear magnitudes from visual observations, such as different defocussing methods (see e.g. Kamél 1991). Though we have taken away all the visual observations from the CLICC data set, it was not possible to do the same for MPC data due to the lack of information. Nevertheless, the number of visual reports of nuclear magnitudes has been very low in recent years.

Though the amateur contribution has been of great value in the analysis of total magnitudes, in particular perihelion comet lightcurves, the quality of the amateur nuclear magnitudes is very poor, which makes their reports of little use, unless the comet is far from the Sun and inactive, but "professional-like" amateurs with large telescopes and CCDs, like William Offutt, are then required.

The data taken from the HST observations included in our catalog correspond to only 4 comets: $4 \mathrm{P} /$ Faye, 22P/Kopff, 9P/Tempel 1 and 46P/Wirtanen. There are observations of $19 \mathrm{P} /$ Borrelly and $45 \mathrm{P} /$ Honda-MrkosPajdušáková, but they were taken at large phase angles $\left(38^{\circ}\right.$ and $90^{\circ}$, respectively). As explained below, these large phase angle observations are discarded from our catalog.

\subsection{The screening procedure and the reduction}

As mentioned, we have discarded all magnitudes determined visually from the CLICC data set since they are too unreliable. We could not do the same for the MPC data, but there are likely very few visual observations there anyway. Total magnitudes of comet 29P/SchwassmannWachmann 1 were also deleted. This comet has a quasicircular orbit with a semimajor axis of 6 AU. The reasons to include total magnitudes at $r>3 \mathrm{AU}$ are not valid in this case because the comet is well known to intermittently exhibit major activity ("outbursts") by an as yet unknown mechanism. It has been well observed all around its orbit; total magnitudes are generally many units brighter than the large set of more than 200 nuclear estimates.

Following Kamél, we have corrected all the photographic magnitudes $(P)$ for color, introducing the color correction: $P-V=0.6$. Concerning red magnitudes, observations show that the coma-subtracted nuclei are slightly redder than the Sun in that part of the spectrum (a gradient of the reflectivity of about $10 \%$ per $1000 \AA$ at optical wavelengths). While the Sun has a color in the 
Kron-Cousins system of $V-R=0.36$, observations of 28P/Neujmin 1, 10P/Tempel 2 and 46P/Wirtanen show an average $V-R \sim 0.5$ (e.g. Jewitt \& Meech 1987; Jewitt \& Luu 1989; Lamy et al. 1998a). Some of the red magnitudes were obtained with the Mould $R$ filter, which is close enough to the Kron-Cousins $R$ magnitude to consider the difference as negligible at the expected level of accuracy. Therefore, we will apply a standard color correction $V-R=0.5$.

From comparison of MPC reports and IAUC (where more information is given on the magnitude estimates), we have seen that the following observers always report magnitudes in $R$ : S. Larson and C. Hergenrother (from many telescopes at Kitt Peak and Whipple Observatory - Mt. Hopkins); D. Jewitt, K. Meech and collaborators (from Mauna Kea); H. Boehnhardt (from ESO); A. Fitzsimmons \& I. Williams (from La Palma). In case no information is provided about the color, we then assume that they report $R$-magnitudes and the proper color correction is applied.

The observed apparent magnitudes $V$ are then transformed into absolute magnitudes $V(1,1,0)$ by:

$V(1,1,0)=V-5 \times \log (r \Delta)-\beta \alpha$

where $r$ and $\Delta$ are the heliocentric and geocentric distances at the instant of the observation and $\alpha$ is the phase angle. $\beta$ is the phase coefficient, for which we have assumed a common value of $0.04 \mathrm{mag} /$ degree for all the comets (Jewitt \& Luu 1989). Since the phase coefficient might depart significantly from the adopted value in individual cases (Scotti, private communication), we have discarded magnitudes measured at phase angles greater than $30^{\circ}$ in order to avoid gross errors in the phase correction.

For each data point we compute ephemeris to calculate the values of $r, \Delta$ and $\alpha$ and apply the correction from apparent to absolute magnitudes.

\section{Plots of $V(1,1,0)$ vs. $r$}

In Appendix B we present plots of $V(1,1,0)$ vs. $r$ for the 138 comets for which data is available (an example is presented in Fig. 2). The vertical lines indicate the perihelion and aphelion distances. Since the comet may have changed these two values during the observed period, we choose the minimum perihelion and maximum aphelion distance in that period of time. Different symbols are assigned to selected observers and observational categories as discussed in Sect. 3.2. The observations are classified using the following groups:

- CCD - observations taken by professional astronomers using medium- and large-sized telescopes (1.5 m aperture or bigger) with CCDs;

- Roemer - E. Roemer and her collaborators' reports of nuclear magnitudes;

- Scotti nuclear - Observations by J. Scotti reported as nuclear magnitudes; we are not able to identify

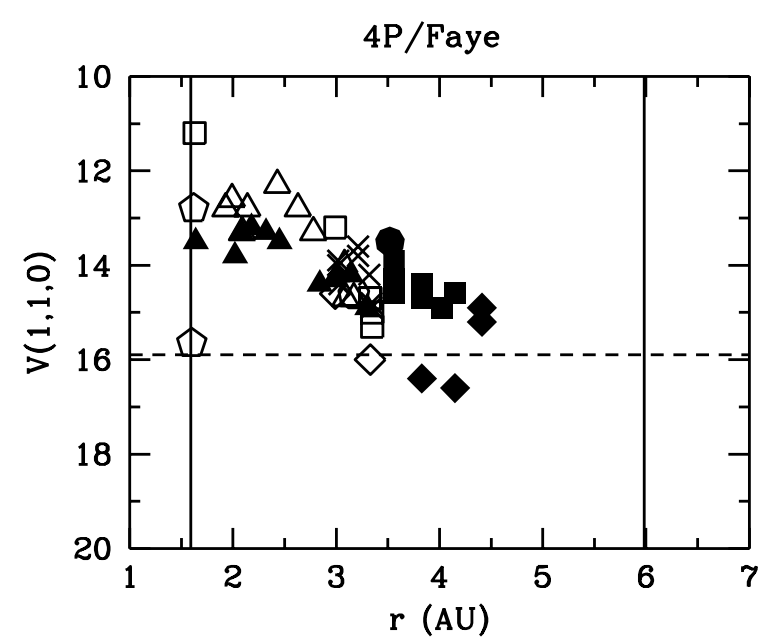

Fig. 2. An example of the Plots of $V(1,1,0)$ vs. $r$ that appear in Appendix B. The symbols have the following correspondence: pre-perihelion - empty; post-perihelion - full; for the different observers we have: CCD - pentagon; Roemer - triangle; Scotti (nuclear) - diamond; Scotti (total) - square; General - circle; Total $r>3 \mathrm{AU}$ - pre - cross, post - chicken-foot

whether the coma subtraction method was applied, though in many cases this is inferred because total and nuclear magnitudes are given for the same date;

- Scotti total - J. Scotti's reports of total magnitudes, including those at $r \leq 3 \mathrm{AU}$;

- General - Nuclear reports by any other observer not included in the previous groups; most of the observers are amateur astronomers;

- Total - Reports of total magnitudes taken at $r>3 \mathrm{AU}$ by any observer except J. Scotti.

\section{Sources of errors and uncertainties}

Several sources of uncertainties can be identified in the determination of the adopted nuclear magnitudes and in the computation of the effective nuclear radius. Let us now analyze these in some detail.

We may broadly distinguish between methodological errors due to shortcomings of the observing techniques and intrinsic errors due to unknown properties of the nuclei themselves. The former include all random and systematic errors of photometry, which are often particularly bad for attempted measurements on cometary nuclei. Some of the reasons are that many of the data were taken for non-photometric purposes where magnitudes resulted as a spinoff without much attention to the photometric conditions of the sky, and that the data often refer to objects near the plate limit without appropriate standard sequences, or uncertainty of the CCD extrapolation.

It must be kept in mind that most of the magnitudes in the literature, even if referred to as nuclear, are not truly nuclear magnitudes according to our physical definition. This is the main concern of our investigation, and our 
most important aim is to minimize these errors, which are due to coma contamination. Nonetheless, the discrepancies remaining between our adopted nuclear magnitudes and the true values have an important component that can be referred to as residual nuclear activity. This component borders between the methodological and intrinsic errors, because:

(1) the angular resolution of the observation has a major influence on the amount of coma contamination for an active comet (methodological error);

(2) even if the best possible angular resolution is achieved, or if the comet appears perfectly inactive, the nucleus is never resolved and the apparent nucleus may always be contaminated by circumnuclear dust (intrinsic error).

To the above errors, we should finally add some further intrinsic errors due to the nucleus itself: variations of the photometric nucleus cross-section $S$ due to rotation; and a phase angle effect. Furthermore, the computation of the nuclear effective radius requires precise knowledge of the geometric albedo, which is generally not available.

\subsection{The adopted nuclear magnitudes}

\subsubsection{The data values}

The error of each individual observed magnitude may be of the order of 0.1 magnitudes or more, except for the case of CCD observations obtained from a few papers where photometry was done carefully; errors as low as a few hundredths of a magnitude can be attained in this case. A few tenths of a magnitude is the usual error of photographic observations. While most CCD magnitudes reported in the MPCs are more indicative values than precise determinations, the great improvement of the recent CCD data with respect to the old photographic data is the huge number of magnitude determinations at larger heliocentric distances. Unfortunately, in many cases the color of the magnitude is not clearly established, or it can only be crudely approximated by a standard color, as occurs, for instance, with Scotti's unfiltered magnitudes.

\subsubsection{Nuclear rotation}

Cometary nuclei seem to be very irregular and elongated objects. Therefore, the photometric nucleus cross-section may vary with the position of the nucleus with respect to the line of sight. If the spin axis of the nucleus is not aligned with the line of sight, rotation produces fast variations of the photometric cross-section $S$. Rotational lightcurves have been used to determine the rotation period of cometary nuclei (see e.g. Jewitt \& Meech 1987). Assuming that the nucleus is a triaxial ellipsoid with axes $a>b>c$, that it has a homogeneous surface, and that its spin is relaxed to pure rotation around the fixed axis $c$, it is easy to see that the maximum amplitude of its rotational lightcurve is

$B_{\mathrm{N} 2} / B_{\mathrm{N} 1}=b / a=10^{-0.4 \Delta m}$

where $B_{\mathrm{N} 1}$ and $B_{\mathrm{N} 2}$ are the maximum and minimum brightnesses. For instance, the lightcurve of a nucleus with $a / b=2$ fulfilling the previous conditions will show a maximum amplitude of 0.75 mag. So, for typical complex shapes, variations up to the order of one magnitude may be observed due only to the rotation of the comet (see e.g. Lamy et al. 1998b).

\subsubsection{Phase coefficient $\beta$}

As regards the phase coefficient $\beta$, Scotti (private communication) has pointed out that it might depart significantly from our adopted value. Since we have taken a limit for the phase angle of $30^{\circ}$, an uncertainty on $\beta$ of \pm 0.02 would introduce a maximum uncertainty of \pm 0.6 in the adopted nuclear magnitude.

The three effects discussed so far imply that, even for a non-active nucleus, a dispersion of the order of at least one magnitude in the plots should be considered as normal.

\subsubsection{Non-detected activity}

Some comets present much larger dispersions or a trend in the plots (see Sect. 8.3) that suggests some non-detected activity. The detection of a small coma, especially when the comet is far from the Earth, is not a simple task. Licandro et al. (1999a) compare the comet brightness profile to the profile of field stars, which proves to be very useful, but even with such methods some faint coma may remain undetected. But for most of the data included in the catalog the presence of a coma is reported only when it is easily detected by eye or, in the best case, by comparing the FWHM of the comet with respect to the one of the stars in CCD images.

The only way to determine if the comet is active with the data of the catalog is to compare the nuclear magnitudes obtained at a wide range of heliocentric distances. If the trend of the data becomes horizontal beyond a certain heliocentric distance, and the dispersion of the data is not large (say, less than one magnitude), we should expect that the corresponding average magnitude will correspond to the one of the bare nucleus. Yet, some residual dust coma might remain at some constant level over the whole observed part of the cometary orbit, even if the comet does not present any signs of activity. The main problem with this uncertainty, with respect to the other ones, is that it introduces a systematic effect that produces brighter "nuclear" magnitudes. 


\subsubsection{Coma subtraction}

A method was already explained in Sect. 3.2 as developed and applied by James Scotti. We showed that it involves in itself several sources of errors and uncertainties.

The HST team also applied a method of coma subtraction to their observations. A narrow PSF and a very good sampling enables them to obtain a more reliable estimate of the nucleus contribution, even in cases similar to the one sketched in Fig. 1a.

\subsection{The computation of the nuclear radius}

Let us also consider the uncertainties in the determination of the effective nuclear radius. Let us consider an uncertainty in the geometric albedo of $\Delta p_{\mathrm{v}}$ and in the absolute magnitude of $\Delta H_{\mathrm{N}}$, leading to a combined uncertainty in the radius of $\Delta R_{\mathrm{N}}$. Applying Eq. (1) to $\left(R_{\mathrm{N}}, H_{\mathrm{N}}, p_{\mathrm{v}}\right)$ and $\left(R_{\mathrm{N}}+\Delta R_{\mathrm{N}}, H_{\mathrm{N}}+\Delta H_{\mathrm{N}}, p_{\mathrm{v}}+\Delta p_{\mathrm{v}}\right)$ and taking the difference, we obtain

$\log \left(1+\frac{\Delta R_{\mathrm{N}}}{R_{\mathrm{N}}}\right)=-1 / 2\left[\log \left(1+\frac{\Delta p_{\mathrm{v}}}{p_{\mathrm{v}}}\right)+0.4 \Delta H_{\mathrm{N}}\right]$.

For example, an uncertainty of $\Delta H_{\mathrm{N}}= \pm 1 \mathrm{mag}$ would lead to a $-37 \% /+58 \%$ relative error in the radius estimate. Adopting a value $p_{\mathrm{v}}=0.04$ and considering an uncertainty of \pm 0.02 , the uncertainty in the radius estimate would be $-44 \% /+78 \%$. Note that, for radius determination, a one magnitude error in the nuclear magnitude is even less important than the uncertainty due to the bad knowledge of cometary albedo. Individual nuclear magnitudes to a precision much better than one magnitude are not needed for a first characterization of the size or mass distribution either. We are thus justified in considering all $H_{\mathrm{N}}$ values with errors within roughly one magnitude as meaningful data.

\section{Defining our "best estimates" for the nuclear magnitudes}

Several criteria have been taken into account when assigning $H_{\mathrm{N}}$ values to the JF comets. Furthermore, we have classified the adopted nuclear magnitudes into four quality classes. We now present some general considerations that were used for $H_{\mathrm{N}}$ assignment and quality classification.

- We require consistency of several observations taken at somewhat different heliocentric distances. In almost all cases, CCD data taken from the Spacewatch or larger telescopes are involved. The cases where such data are absent are confined to the worst quality class or eliminated altogether;

- In many cases, different observers give discordant magnitudes. Typically, a set of corrected nuclear magnitudes by Scotti is fainter than another set of data.
The weight given to the Scotti data then depends on the number of his observations, their spacing in $r$, and their internal consistency. A single observation is usually discarded, a couple of closely spaced observations is given low weight, while if there are three or more data points well spaced in $r$ and in good mutual agreement we give to their mean a strong weight in the derived magnitude;

- We take special care of comets that show a strong trend for the nuclear magnitudes to get fainter with increasing $r$. If there is not a clear indication of a leveling off and if the observations do not approach the aphelion distance, the quality class assigned is usually the worst. The adopted magnitude is a mean of the faintest, more distant observations, and this should be taken as an upper limit to the true magnitude of the comet nucleus;

- Total magnitudes at $r>3$ AU are included for purposes of comparison with nuclear magnitudes measured at similar distances. If the total magnitudes are much brighter than the nuclear ones, this is an indication that the comet keeps active all along the orbit. On the other hand, if the total magnitudes are similar to the nuclear ones, the comet may have little or no activity;

- In line with our choice of not placing much weight on single observations or close pairs, whether discordant or consistent, even if these data are of the CCD kind, we have discarded almost all comets where the only data were such. The only comet included in this category was $97 \mathrm{P} /$ Metcalf-Brewington, where the observations were taken at far heliocentric distances and the comet was inactive (Licandro et al. 1999a).

Our definition of the quality classes (QC) is as follows:

QC 1: These are our best nuclear magnitudes for which we have many observations spread along a wide range of heliocentric distances and from more than one observer. The uncertainty in the adopted nuclear magnitude is less than or about \pm 0.3 mag.

QC 2: These are fairly good estimates based on several observations spread throughout a wide range of $r$, or few observations but made at very large $r$. The uncertainty is estimated to be between $\sim \pm 0.3$ and \pm 0.6 mag.

QC 3: These are estimates made at different $r$ with a somewhat larger scatter than for the previous class. The uncertainty should be between $\sim \pm 0.6$ and \pm 1 mag.

QC 4: These are poor estimates, either because they rely upon old data, or upon very few observations, or because the observations show a large scatter. Some of them can only be considered as a lower limit to the nuclear magnitude (i.e., the true magnitude of the comet nucleus is fainter than the quoted value). The uncertainty is generally well above \pm 1 mag. 


\section{The adopted nuclear magnitudes and radii}

From the analysis of the 138 plots shown in Appendix B we assign nuclear magnitudes to 105 JF comets, out of which 9 belong to $\mathrm{QC} 1,18$ to $\mathrm{QC} 2,37$ to $\mathrm{QC} 3$, and 41 to QC 4. We discarded 33 JF comets, for which we were not able to adopt or even approach a reasonable estimate of a nuclear magnitude, either because there are very few data (in many cases a single nuclear magnitude), or because a major coma contamination in the reported magnitudes is strongly suspected. This is usually the case for data coming from some observers who systematically report nuclear magnitudes too bright as compared with other reports. The discarded JF comets are shown in Table 3.

There are in addition $27 \mathrm{JF}$ comets discovered through 1997 with no plots, because they do not have any reported nuclear magnitudes. Some of these JF comets are very old and long ago ceased to be observed (a few of them have already disappeared like 3D/Biela), and some are very recent discoveries, observed only at one apparition with no chance of obtaining nuclear magnitudes. The JF comets of Marsden \& Williams' (1997) catalogue that appear neither in Table 3 nor in the plots of Appendix B are shown in Table 4.

Table 5 contains the list of our $105 \mathrm{JF}$ comets with assigned nuclear magnitudes. We present: 1) name of the comet, 2) perihelion distance, 3) largest heliocentric distance at which a nuclear magnitude was observed, 4) number of perihelion passages during which the comet was observed, 5) adopted absolute nuclear magnitude, 6) quality class, and 7) effective nuclear radius assuming a geometric albedo $p_{\mathrm{v}}=0.04$.

\section{Discussion}

\subsection{Reliability of our adopted magnitudes}

To discuss the reliability of our adopted nuclear magnitudes, we performed a few tests by comparing different subsets of data. In Figs. $3 \mathrm{a}$ and $3 \mathrm{~b}$ we compare the adopted nuclear magnitudes with respect to different quality classes. We group the comets of QC 1, 2 and 3 together, and compare them with those of QC 4 . We find just minor differences between the two subsets. There are slightly more faint magnitudes in QC 4 than in the rest. This is consistent with the expectation in case the magnitudes are generally free of systematic errors. The only correlation in such a case should be due to the smallest nuclei being more difficult to observe and hence showing a preference for QC 4, just as we observe.

In Figs. 4a and 4b we use as a classification criterion the number of revolutions spanned by the observations. We distinguish two groups: comets that have been observed during several revolutions, and those observed at a)

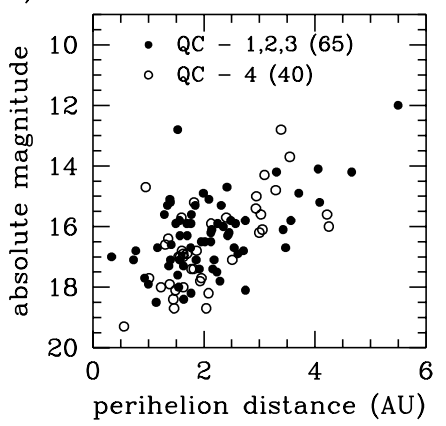

b)

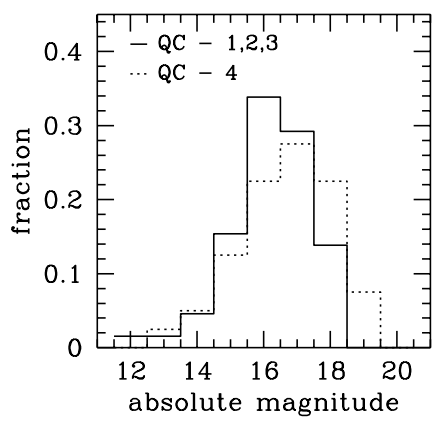

Fig. 3. a) Adopted nuclear magnitudes vs. perihelion distance for comets in QC 1-3 (full circles) and comets in QC 4 (open circles), the numbers in parentheses correspond to the numbers of objects in the quality classes. b) The marginal distributions of magnitudes for the subsets shown in $3 \mathrm{a}$

just one perihelion passage. While the marginal distribution in Fig. 4b indicates a trend for brighter nuclei of oneapparition comets, one easily sees from Fig. 4a that this is due to a preference of larger perihelion distances of oneapparition comets. This is, in turn, due to the fact that large- $q$ comets have a much shorter history of discoveries than their small- $q$ analogues.

Focussing on a range of $q \lesssim 2.5 \mathrm{AU}$, where both classes of comets are well represented, one sees no significant difference between their $H_{\mathrm{N}}$ distributions. Had such a difference appeared, this would have led to some worry over the quality of our magnitudes, especially if the one-apparition comets had shown brighter nuclei. It is true that these are often active near aphelion (see further Sect. 8.3), but apparently our $H_{\mathrm{N}}$ values are not seriously affected by this. Nonetheless, we generally give a low quality class to the adopted nuclear magnitude based on data taken on one passage; there is a larger fraction of comets with QC 4 compared to the ones with QC 1-3 in the set of onepassage comets $(13: 10)$ than in the set with more than one passage (28:54).

In the following test we use as a classification criteria the activity at large heliocentric distances (Figs. 5a and 5b). In Sect. 8.3 we explain the parameters used to classify the comets according to the activity. From the comparison of the two marginal distribution in Fig. $5 \mathrm{~b}$ we note a high fraction of active comets at $H_{\mathrm{N}} \sim 16$. This value is mainly due to the cluster of comets at $q \sim 4$ AU seen in Fig. 5a. As in the previous test, focussing on a range of $q \lesssim 2.5 \mathrm{AU}$ one sees no significant difference between their $H_{\mathrm{N}}$ distributions.

\subsection{Comparisons among observers}

The differences between the reported nuclear magnitudes and our best estimates are plotted in Fig. 6 as a function of the heliocentric distances of the observations, for the data of Roemer's, CCD (general), and Scotti's nuclear 
Table 3. Discarded JF comets

\begin{tabular}{lll}
\hline 85P/Boethin & 140P/Bowell-Skiff & 108P/Ciffréo \\
54P/de Vico-Swift & 79P/du Toit-Hartley & D/1978R1 (Haneda-Campos) \\
D/1952B1 (Harrington-Wilson) & $\mathrm{P} / 1987 \mathrm{Q} 3$ (Helin) & 127P/Holt-Olmstead \\
59P/Kearns-Kwee & $\mathrm{P} /$ 1997B1 (Kobayashi) & 104P/Kowal 2 \\
$\mathrm{P} / 1996 \mathrm{R} 2$ (Lagerkvist) & $\mathrm{P} /$ 1997T3 (Lagerkvist-Carsenty) & 93P/Lovas 1 \\
$\mathrm{P} / 1986 \mathrm{~W} 1$ (Lovas 2) & 115P/Maury & 18D/Perrine-Mrkos \\
80P/Peters-Hartley & 83P/Russell 1 & 92P/Sanguin \\
106P/Schuster & 102P/Shoemaker 1 & $\mathrm{P} / 1986 \mathrm{~A} 1$ (Shoemaker 3) \\
121P/Shoemaker-Holt 2 & 129P/Shoemaker-Levy 3 & $\mathrm{P} / 1991 \mathrm{~T} 1$ (Shoemaker-Levy 5) \\
D/1977C1 (Skiff-Kosai) & 69P/Taylor & D/1978C2 (Tritton) \\
D/1960S1 (van Houten) & 63P/Wild 1 & 114P/Wiseman-Skiff \\
\hline
\end{tabular}

Table 4. JF comets without observed nuclear magnitudes

\begin{tabular}{lll}
\hline D/1884O1 (Barnard 1) & D/1892T1 (Barnard 3) & 3D/Biela \\
D/1819W1 (Blanpain) & D/1886K1 (Brooks 1) & 5D/Brorsen \\
D/1894F1 (Denning) & 72P/Denning-Fujikawa & 66P/Du Toit \\
34D/Gale & P/1988V1 (Ge-Wang) & D/1896R2 (Giacobini) \\
D/1766G1 (Helfenzrieder) & D/1984H1 (Kowal-Mrkos) & D/1770L1 (Lexell) \\
P/1994P1-A (Machholz 2) & P/1992G3 (Mueller 4) & 25D/Neujmin 2 \\
D/1783W1 (Pigott) & D/1984W1 (Shoemaker 2) & P/1990V1 (Shoemaker-Levy 1) \\
P/1991V1 (Shoemaker-Levy 6) & D/1993F2 (Shoemaker-Levy 9) & D/1918W1 (Schorr) \\
D/1895Q1 (Swift) & 11D/Tempel-Swift & 107P/Wilson-Harrington \\
\hline
\end{tabular}

a)

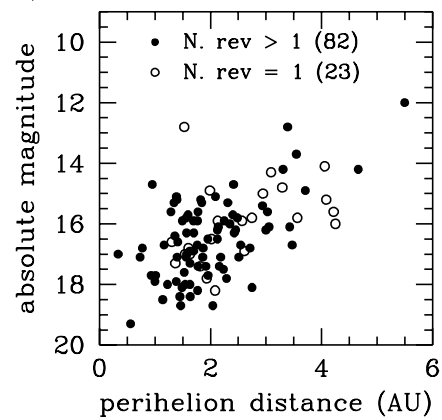

b)

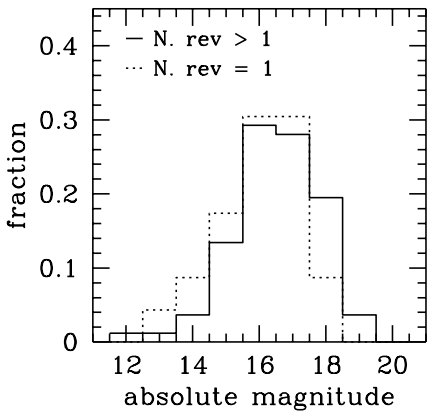

Fig. 4. a) Adopted nuclear magnitudes vs. perihelion distance for comets with observations spanning more than one revolution (full circles) and observed just in the discovery passage (open circles), the numbers in parentheses correspond to the numbers of objects in the defined groups. b) The marginal distributions of magnitudes for the subsets shown in $4 \mathrm{a}$

magnitudes (magnitudes from Scotti reported as total are not considered). Each observation is represented as a dot. The stars correspond to the mean value in bins of $1 \mathrm{AU}$ in heliocentric distance.

Roemer's data is usually about two magnitudes brighter than our best estimates of the nuclear magnitudes (see Fig. 6a) at any heliocentric distance. This is a clear indication that Roemer's magnitudes are strongly affected by coma contamination. The observations closer to the Sun show a large scatter with a trend to increase the difference between Roemer's and our values, presumably due to the larger activity of comets closer to the Sun. a)

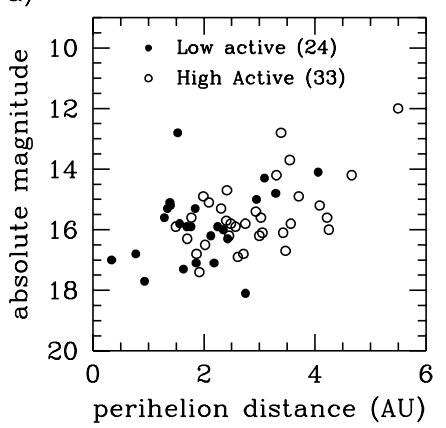

b)

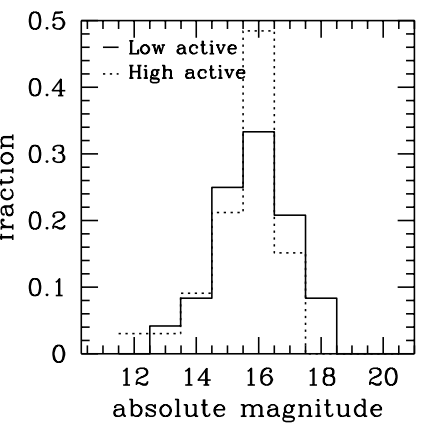

Fig. 5. a) Adopted nuclear magnitudes vs. perihelion distance for comets with low activity at large heliocentric distances (classes L and I in Sect. 8.3) (full circles) and comets with high activity (classes $\mathrm{H}$ and $\mathrm{V}$ ) (open circles), the numbers in parentheses correspond to the numbers of objects in the defined groups. b) The marginal distributions of magnitudes for the subsets shown in $5 \mathrm{a}$

We conclude that Roemer's data provide good values of the nuclear magnitudes only for low active comets, so all the conclusions about nuclear magnitudes of JF comets and masses derived from her data (e.g. Shoemaker \& Wolfe 1982), should be revisited (see Fernández et al. 1999).

CCD data have strong signs of coma contamination in most of the observations done at $r<4$ AU (see Fig. 6b). The scatter of the observations at $r>4$ AU (except the data points at $r \sim 6 \mathrm{AU}$, see below), and those that give fainter values than our estimated magnitudes can be 
Table 5. Absolute nuclear magnitudes and radii

\begin{tabular}{|c|c|c|c|c|c|c|}
\hline Comet & $q(\mathrm{AU})$ & $r_{\max }(\mathrm{AU})$ & N. pas. & $H_{\mathrm{N}}$ & $\mathrm{QC}$ & $R(\mathrm{~km})$ \\
\hline 50P/Arend & 1.821 & 2.50 & 4 & 15.2 & 4 & 3.0 \\
\hline 49P/Arend-Rigaux & 1.386 & 4.79 & 8 & 15.1 & 1 & 3.2 \\
\hline 47P/Ashbrook-Jackson & 2.311 & 5.33 & 7 & 15.3 & 1 & 2.9 \\
\hline 19P/Borrelly & 1.395 & 5.62 & 5 & 15.2 & 1 & 3.0 \\
\hline P/1992Q1 (Brewington) & 1.601 & 3.26 & 1 & 16.8 & 4 & 1.5 \\
\hline 16P/Brooks 2 & 1.950 & 2.66 & 5 & 16.5 & 3 & 1.7 \\
\hline $87 \mathrm{P} / \mathrm{Bus}$ & 2.183 & 3.78 & 2 & 17.1 & 3 & 1.3 \\
\hline 101P/Chernykh & 2.568 & 4.17 & 1 & 15.9 & 3 & 2.2 \\
\hline 67P/Churyumov-Gerasimenko & 1.285 & 4.98 & 4 & 15.6 & 1 & 2.5 \\
\hline $71 \mathrm{P} /$ Clark & 1.560 & 4.01 & 4 & 17.1 & 2 & 1.3 \\
\hline 32P/Comas-Sola & 1.772 & 3.87 & 6 & 15.6 & 3 & 2.5 \\
\hline 33P/Daniel & 1.382 & 2.87 & 3 & 17.9 & 4 & 0.9 \\
\hline 6P/D'Arrest & 1.163 & 4.86 & 5 & 16.7 & 2 & 1.5 \\
\hline 57P/Du-Toit-Neujmin-Delporte & 1.305 & 3.29 & 1 & 16.6 & 4 & 1.6 \\
\hline $2 \mathrm{P} /$ Encke & 0.336 & 4.09 & 11 & 17.0 & 3 & 1.3 \\
\hline 4P/Faye & 1.692 & 4.41 & 7 & 15.9 & 2 & 2.2 \\
\hline 15P/Finlay & 0.998 & 3.65 & 3 & 17.9 & 4 & 0.9 \\
\hline $37 \mathrm{P} /$ Forbes & 1.528 & 3.59 & 5 & 17.6 & 3 & 1.0 \\
\hline 90P/Gehrels 1 & 2.935 & 3.35 & 2 & 15.4 & 4 & 2.8 \\
\hline $78 \mathrm{P} /$ Gehrels 2 & 2.348 & 3.70 & 3 & 16.0 & 3 & 2.1 \\
\hline $82 \mathrm{P} /$ Gehrels 3 & 3.424 & 4.08 & 3 & 16.1 & 2 & 2.0 \\
\hline P/1997C1 (Gehrels) & 3.565 & 5.45 & 1 & 15.8 & 3 & 2.3 \\
\hline 21P/Giacobini-Zinner & 0.931 & 4.83 & 7 & 17.7 & 2 & 1.0 \\
\hline 84P/Giclas & 1.627 & 2.18 & 2 & 16.9 & 4 & 1.4 \\
\hline 26P/Grigg-Skjellerup & 0.732 & 4.93 & 4 & 17.1 & 1 & 1.3 \\
\hline $65 \mathrm{P} / \mathrm{Gunn}$ & 3.306 & 4.73 & 7 & 14.2 & 2 & 4.8 \\
\hline 51P/Harrington & 1.694 & 2.35 & 3 & 16.9 & 4 & 1.4 \\
\hline $52 \mathrm{P} /$ Harrington-Abell & 1.774 & 3.02 & 7 & 17.4 & 4 & 1.1 \\
\hline 100P/Hartley 1 & 1.540 & 1.86 & 1 & 17.0 & 4 & 1.3 \\
\hline 103P/Hartley 2 & 0.952 & 4.73 & 3 & 14.7 & 4 & 3.8 \\
\hline 110P/Hartley 3 & 2.454 & 4.64 & 3 & 16.2 & 2 & 1.9 \\
\hline P/1993K2 (Helin-Lawrence) & 3.090 & 5.02 & 1 & 14.3 & 4 & 4.6 \\
\hline 117P/Helin-Roman-Alu 1 & 3.707 & 5.21 & 2 & 14.9 & 2 & 3.5 \\
\hline 132P/Helin-Roman-Alu 2 & 1.930 & 1.97 & 1 & 17.8 & 4 & 0.9 \\
\hline 111P/Helin-Roman-Crockett & 3.470 & 4.54 & 2 & 16.7 & 3 & 1.5 \\
\hline 17P/Holmes & 2.141 & 3.25 & 5 & 16.1 & 3 & 2.0 \\
\hline 45P/Honda-Mrkos-Pajdušáková & 0.559 & 1.95 & 2 & 19.3 & 4 & 0.5 \\
\hline 88P/Howell & 1.916 & 3.29 & 4 & 17.4 & 3 & 1.1 \\
\hline 58P/Jackson-Neujmin & 1.463 & 2.06 & 3 & 18.7 & 4 & 0.6 \\
\hline P/1995A1 (Jedicke) & 4.083 & 5.53 & 1 & 15.2 & 3 & 3.0 \\
\hline P/1996A1 (Jedicke) & 4.055 & 6.52 & 1 & 14.1 & 2 & 5.0 \\
\hline 48P/Johnson & 2.248 & 3.98 & 6 & 15.9 & 2 & 2.2 \\
\hline 68P/Klemola & 1.763 & 3.87 & 3 & 15.9 & 3 & 2.2 \\
\hline 75P/Kohoutek & 1.568 & 3.82 & 3 & 16.3 & 3 & 1.8 \\
\hline 70P/Kojima & 1.631 & 3.77 & 3 & 17.3 & 3 & 1.2 \\
\hline 22P/Kopff & 1.699 & 4.72 & 9 & 16.3 & 2 & 1.8 \\
\hline 99P/Kowal 1 & 4.660 & 6.12 & 2 & 14.2 & 3 & 4.8 \\
\hline 134P/Kowal-Vávrová & 2.609 & 3.70 & 1 & 16.9 & 3 & 1.4 \\
\hline P/1994A1 (Kushida) & 1.367 & 2.55 & 1 & 17.3 & 3 & 1.2 \\
\hline P/1993X1 (Kushida-Muramatsu) & 2.745 & 3.81 & 1 & 15.8 & 3 & 2.3 \\
\hline P/1997V1 (Larsen) & 3.293 & 3.40 & 1 & 14.8 & 4 & 3.6 \\
\hline 77P/Longmore & 2.402 & 4.09 & 4 & 15.7 & 4 & 2.4 \\
\hline 130P/McNaught-Hughes & 2.125 & 4.00 & 2 & 16.5 & 3 & 1.7 \\
\hline 97P/Metcalf-Brewington & 1.631 & 3.67 & 1 & 17.0 & 4 & 1.3 \\
\hline P/1997G1 (Montani) & 4.217 & 4.80 & 1 & 15.6 & 4 & 2.5 \\
\hline $124 \mathrm{P} /$ Mrkos & 1.411 & 3.58 & 2 & 16.6 & 2 & 1.6 \\
\hline 120P/Mueller 1 & 2.747 & 3.15 & 2 & 18.1 & 3 & 0.8 \\
\hline
\end{tabular}


Table 5. continued

\begin{tabular}{|c|c|c|c|c|c|c|}
\hline Comet & $q(\mathrm{AU})$ & $r_{\max }(\mathrm{AU})$ & N. pas. & $H_{\mathrm{N}}$ & $\mathrm{QC}$ & $R(\mathrm{~km})$ \\
\hline 131P/Mueller 2 & 2.083 & 2.44 & 1 & 18.2 & 4 & 0.8 \\
\hline 136P/Mueller 3 & 2.998 & 3.38 & 2 & 16.2 & 4 & 1.9 \\
\hline P/1993W1 (Mueller 5) & 4.250 & 4.95 & 1 & 16.0 & 4 & 2.1 \\
\hline $28 \mathrm{P} /$ Neujmin 1 & 1.529 & 8.97 & 1 & 12.8 & 1 & 9.1 \\
\hline $42 \mathrm{P} /$ Neujmin 3 & 2.042 & 2.52 & 3 & 18.7 & 4 & 0.6 \\
\hline 39P/Oterma & 3.389 & 4.54 & 2 & 12.8 & 4 & 9.1 \\
\hline 119P/Parker-Hartley & 3.026 & 4.18 & 2 & 15.6 & 4 & 2.5 \\
\hline 7P/Pons-Winnecke & 0.772 & 3.55 & 5 & 16.8 & 3 & 1.5 \\
\hline 30P/Reinmuth 1 & 1.860 & 3.39 & 6 & 17.1 & 3 & 1.3 \\
\hline 44P/Reinmuth 2 & 1.867 & 4.09 & 6 & 16.8 & 4 & 1.5 \\
\hline 89P/Russell 2 & 2.159 & 3.07 & 2 & 17.4 & 3 & 1.1 \\
\hline 91P/Russell 3 & 2.510 & 3.73 & 3 & 17.1 & 4 & 1.3 \\
\hline 94P/Russell 4 & 2.125 & 3.54 & 2 & 16.2 & 3 & 1.9 \\
\hline $24 \mathrm{P} /$ Schaumasse & 1.226 & 2.75 & 4 & 18.0 & 4 & 0.8 \\
\hline 29P/Schwassmann-Wachmann 1 & 5.495 & 7.26 & 4 & 12.0 & 2 & 13.2 \\
\hline 31P/Schwassmann-Wachmann 2 & 2.090 & 4.59 & 7 & 15.1 & 1 & 3.2 \\
\hline 73P/Schwassmann-Wachmann 3 & 1.011 & 3.03 & 2 & 17.7 & 4 & 1.0 \\
\hline 61P/Shajn-Schaldach & 2.234 & 2.84 & 3 & 17.5 & 3 & 1.1 \\
\hline P/1994J3 (Shoemaker 4) & 2.944 & 3.14 & 1 & 15.0 & 4 & 3.3 \\
\hline 128P/Shoemaker-Holt 1 & 3.053 & 3.77 & 2 & 16.1 & 4 & 2.0 \\
\hline 137P/Shoemaker-Levy 2 & 1.844 & 4.84 & 2 & 15.3 & 2 & 2.9 \\
\hline 118P/Shoemaker-Levy 4 & 2.019 & 3.96 & 1 & 16.5 & 3 & 1.7 \\
\hline 138P/Shoemaker-Levy 7 & 1.630 & 1.75 & 2 & 18.0 & 4 & 0.8 \\
\hline 135P/Shoemaker-Levy 8 & 2.711 & 4.14 & 2 & 16.8 & 3 & 1.5 \\
\hline 105P/Singer-Brewster & 1.955 & 3.02 & 2 & 17.7 & 4 & 1.0 \\
\hline 56P/Slaughter-Burnham & 2.544 & 3.66 & 3 & 16.7 & 3 & 1.5 \\
\hline 74P/Smirnova-Chernykh & 3.546 & 4.78 & 4 & 13.7 & 4 & 6.0 \\
\hline 125P/Spacewatch & 1.544 & 3.03 & 2 & 18.0 & 2 & 0.8 \\
\hline 113P/Spitaler & 1.816 & 2.25 & 1 & 17.4 & 4 & 1.1 \\
\hline 64P/Swift-Gehrels & 1.358 & 3.63 & 3 & 16.4 & 4 & 1.7 \\
\hline 98P/Takamizawa & 1.595 & 3.78 & 2 & 15.7 & 4 & 2.4 \\
\hline 9P/Tempel 1 & 1.562 & 4.69 & 6 & 15.8 & 1 & 2.3 \\
\hline 10P/Tempel 2 & 1.344 & 4.74 & 9 & 15.3 & 2 & 2.9 \\
\hline $62 \mathrm{P} /$ Tsuchinshan 1 & 1.486 & 2.37 & 2 & 18.1 & 4 & 0.8 \\
\hline $60 \mathrm{P} /$ Tsuchinshan 2 & 1.769 & 2.31 & 4 & 18.2 & 3 & 0.8 \\
\hline 41P/Tuttle-Giacobini-Kresák & 1.140 & 2.32 & 4 & 18.5 & 3 & 0.7 \\
\hline 112P/Urata-Niijima & 1.449 & 2.87 & 2 & 18.4 & 4 & 0.7 \\
\hline 40P/Väisälä 1 & 1.762 & 3.03 & 3 & 16.7 & 3 & 1.5 \\
\hline 53P/Van Biesbroeck & 2.414 & 6.04 & 4 & 14.7 & 3 & 3.8 \\
\hline 123P/West-Hartley & 2.129 & 2.75 & 1 & 15.9 & 4 & 2.2 \\
\hline 76P/West-Kohoutek-Ikemura & 1.398 & 3.60 & 3 & 17.1 & 3 & 1.3 \\
\hline 36P/Whipple & 2.484 & 4.12 & 6 & 15.8 & 2 & 2.3 \\
\hline $81 \mathrm{P} /$ Wild 2 & 1.491 & 4.42 & 2 & 15.9 & 3 & 2.2 \\
\hline 86P/Wild 3 & 2.288 & 3.43 & 3 & 17.8 & 3 & 0.9 \\
\hline 116P/Wild 4 & 1.989 & 4.14 & 1 & 14.9 & 3 & 3.5 \\
\hline 46P/Wirtanen & 1.635 & 4.62 & 4 & 18.4 & 1 & 0.7 \\
\hline $14 \mathrm{P} /$ Wolf & 1.572 & 3.14 & 6 & 17.0 & 4 & 1.3 \\
\hline 43P/Wolf-Harrington & 2.428 & 3.87 & 7 & 16.3 & 2 & 1.8 \\
\hline
\end{tabular}

explained as due to nucleus rotation and/or errors involved in the computation of the nuclear magnitude (error in the $\beta$ coefficient, color correction, etc.).

Scotti's nuclear magnitudes have to be treated with special care as most of these data are obtained by subtracting the coma contribution (see Sect. 3.2), and the validity of this method should be studied. Figure $6 \mathrm{c}$ shows a large scatter of the data at any heliocentric distance. The interpretation in this case is different from the case of Roemer or CCD data. The scatter in Scotti's data is not due to coma contamination as the method subtracts, in principle, the coma contribution. This scatter may be interpreted as an error induced by the subtraction method (cf. Sect. 3.2). To study the internal consistency of Scotti's 


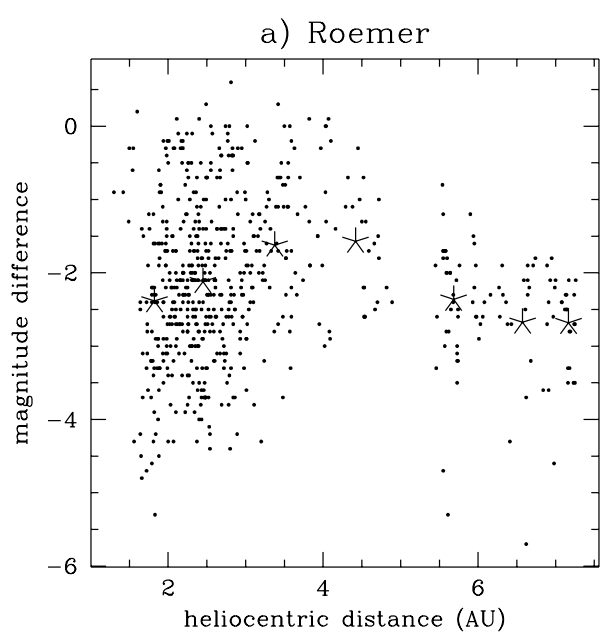

b) $\mathrm{CCD}$

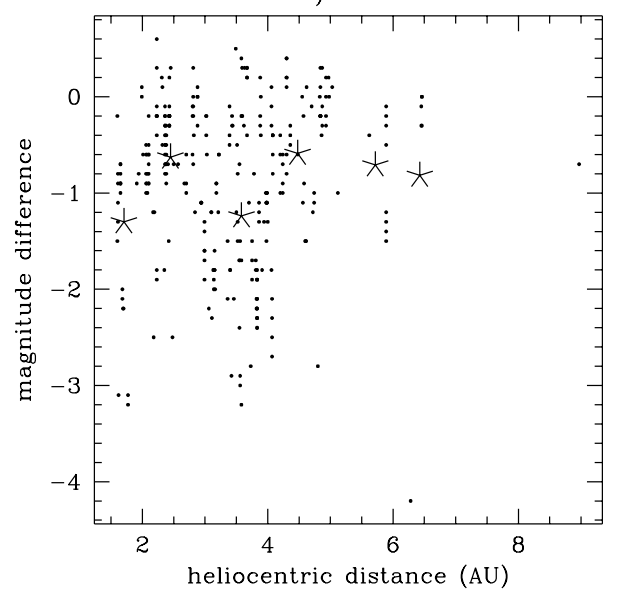

c) Scotti

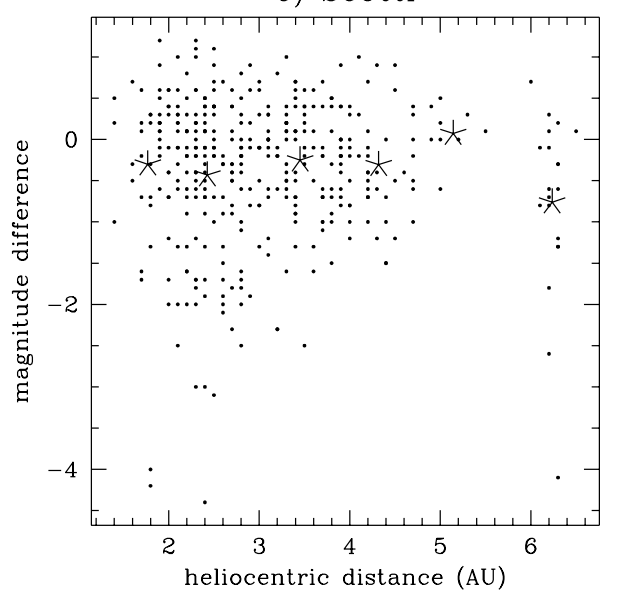

Fig. 6. Comparison between a) Roemer, b) CCD and c) Scotti observations with our adopted magnitudes. The stars correspond to the mean value in bins of $1 \mathrm{AU}$ in heliocentric distance

method, we compute the mean value and the standard deviation $(\sigma)$ of his determinations for all comets with more than three observations (see Fig. 7). Also the difference between Scotti's mean value and our adopted nuclear
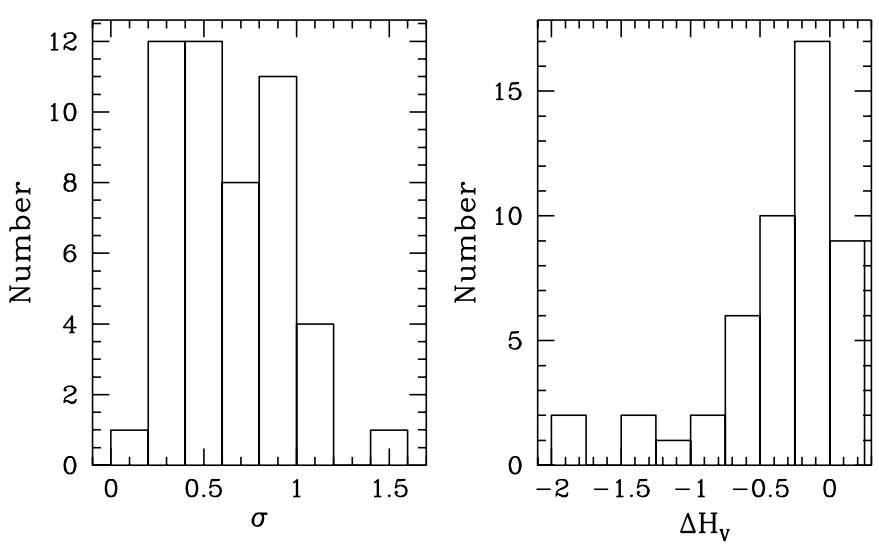

Fig. 7. Histograms of a) standard deviation $(\sigma)$ of Scotti's nuclear magnitude estimates for the same comet, and b) $\Delta H_{V}-$ the difference of the mean of his magnitudes and our adopted nuclear magnitude

magnitude is computed. The mean $\sigma$ value is 0.6 magnitudes, and the largest $\sigma$ is 1.4 magnitudes. In most cases the observations of the same comet obtained at different heliocentric distances give coherent values with an error of some tenths of magnitude, but for some comets their magnitudes show a trend parallel to the one of the "coma unsubtracted" magnitudes (e.g. comets 51P/Harrington, 110P/Hartley 3, 120P/Mueller 1, 24P/Schaumasse, and 9P/Tempel 1). Nevertheless, for most comets the method provides a reasonably good value with an error of some tenths of a magnitude, and it becomes very useful - and meaningful - when several determinations at different heliocentric distances can be collected and compared.

Scotti and CCD values give similar results for comets observed at $r>4 \mathrm{AU}$, within the error bars. The set of points at $r \sim 6 \mathrm{AU}$ in both data sets mainly corresponds to observations of $29 \mathrm{P} /$ Schwassmann-Wachmann 1. This comet shows continuous activity with sporadic outbursts (Jewitt 1990). Note that those magnitudes were either reported as nuclear or a coma subtraction method was applied. The large scatter in the data indicates the difficulties of detecting a faint coma at large heliocentric distances.

Considering that at small $r$ CCD determinations are largely affected by coma contamination, Scotti's data at small $r$ become decisive to estimate the nuclear magnitudes of those comets without observations at $r \gtrsim 4 \mathrm{AU}$, and permits to analyse if the comet is still active at large heliocentric distances by comparing the results with CCD observations.

In Table 6 we compare the HST radius estimates with our own values. Note that we have included comets 19P/Borrelly and 45P/Honda-Mrkos-Pajdušáková that were observed at large phase angles, although they were not included in our data set (i.e. our estimates do not take into account the HST observations for these objects). Taken into account that for our adopted magnitude we include data from many other observers, our estimates 
Table 6. Comparison between the HST and our own comet radius estimates

\begin{tabular}{lcccc}
\hline Comet & $\begin{array}{c}\text { phase } \\
(\mathrm{deg})\end{array}$ & $\begin{array}{c}\text { HST } \\
\text { radius }(\mathrm{km})\end{array}$ & $\begin{array}{c}\text { Our } \\
\text { radius }(\mathrm{km})\end{array}$ & reference \\
\hline 19P/Borrelly & 38 & $1.8-4.4$ & 3.0 & Lamy et al. 1998b \\
45P/Honda-Mrkos-Pajdušáková & 90 & 0.35 & 0.5 & Lamy et al. 1997 \\
22P/Kopff & 3 & $1.65 \mathrm{rr}-1.92$ & 1.8 & Lamy et al. 1996 \\
4P/Faye & $6 \mathrm{r}-19$ & 2.7 & 2.2 & Lamy \& Toth 1995 \\
46P/Wirtanen & 10 & 0.60 & 0.7 & Lamy et al. 1998a \\
9P/Tempel 1 & 4 & $2.8 \mathrm{r}-\mathrm{r} 3.9$ & 2.9 & IAUC 7000 \\
\hline
\end{tabular}

show good agreement with the HST observations, including the cases of the comets observed at very large phase angles.

\subsection{Activity at large heliocentric distances}

Activity at large heliocentric distances $(r>4 \mathrm{AU})$ is not a rare phenomenon. Licandro et al. (1999a) present observations of 18 comets, and find that six of them, observed at $r>4 \mathrm{AU}$, had coma and even tail. Another indication of this kind of activity and the problems to detect it can be observed in the comets that present a trend to increase the estimate of the nuclear brightness with the heliocentric distance, like comets: 2P/Encke, 26P/Grigg-Skjellerup, 103P/Hartley 2, and 10P/Tempel 2. This trend, opposite to the rest of the comets and to the common sense, can only be explained if some remaining activity can be masked in a stellar-like brightness profile.

The data compiled in the present paper can be used to analyse the activity level of the well observed comets. From the plots of reduced magnitudes vs. heliocentric distances $(r)$ shown in Appendix B, we can classify the comets with respect to the degree of activity at large $r$ that can be read off from the dispersion of the brightness observed at large $r$.

Considering all the observations done at $r>3 \mathrm{AU}$ (total and nuclear estimates) we classify the comets with more than 10 observations into four classes defined as follows: class $\mathrm{V}$ (very high activity) if the standard deviation $(\sigma)$ of the data is greater than $2.0 \mathrm{mag}$, class $\mathrm{H}$ (high activity) if the standard deviation $1.0 \leq \sigma<2.0 \mathrm{mag}$; class I (intermediate activity) if $0.4<\sigma \leq 1.0 \mathrm{mag}$; class L (low or vanishing activity) if $\sigma \leq 0.4 \mathrm{mag}$. The limit of $0.4 \mathrm{mag}$ was chosen because for a nucleus of axial ratio $a / b=2 \mathrm{ob}-$ served with its spin axis perpendicular to the line of sight, the typical lightcurve half-amplitude just due to rotation is $\sim 0.4$ magnitudes. Table 7 contains the classes ascribed to a total of 61 comets. Nearly $60 \%$ of the comets are of class $\mathrm{H}$ or $\mathrm{V}$. The explanation for this predominance of remote activity is likely to involve several mechanisms:

- $\mathrm{H}_{2} \mathrm{O}$ sublimation beyond its "standard" limit due to favorably oriented active spots and spin axis near the orbital plane;

- Subsurface sublimation of volatiles like $\mathrm{CO}$ and $\mathrm{CO}_{2}$ due to release of energy from crystallization of amorphous ice at shallow depth;
- Splitting and exposure of fresh ices;

- Reactivation of a comet due to a downward jump in perihelion distance.

Licandro et al. (1999a) found a strong correlation between activity at large heliocentric distances and recent downward jumps in perihelion distances. They relate this correlation to the hypothesis of mantle formation. The large grains cannot be lifted by the outflowing gases and thus remain on the nucleus forming a crust of dust. If the comet remains at a given perihelion distance for a few revolutions, a thin crust is formed that chokes off the sublimation of the ices (Rickman et al. 1990). When a downward jump occurs, the temperature rises, the vapour pressure increases, and the marginally stable crust is blown off. Large areas of fresh ices are then exposed to the Sun; the gas flux, and consequently the dust flux, increases.

Information related to the recent downward jumps is also presented in Table 7, obtained from an updated version of the numerical integrations of the dynamical evolution of Jupiter family comets (Tancredi \& Rickman 1992). These data are graphically presented in Fig. 8. The maximum relative change in the perihelion distance $(\delta q / q)$ experienced by the comet during the last 20 revolutions is plotted against the minimum perihelion distance reached by the comet in the observed period, for the three classes defined above. As expected, there is a strong concentration of low-active JF comets toward $\delta q \sim 0$. By contrast, decreases in perihelion distance $(\delta q<0)$ are usually associated with moderate- to high-active comets. The fact that some JF comets with $\delta q \sim 0$ are rather active is an indication that other factors, besides reduction in $q$, are at work in producing an enhancement in the cometary activity as, for instance, splittings.

\section{Conclusions}

This catalog represents the first comprehensive compilation of nuclear magnitudes of JF comets from different sources. Previous work on this subject was scanty and referred exclusively to the material collected by a few prolific observers, as for instance Roemer or Jewitt. As an example, Shoemaker \& Wolfe (1982) derived the luminosity function of JF comets based only on Roemer's data. A comparison with their results is presented elsewhere (Fernández et al. 1999). 
Table 7. Classification of comets with regard to the degree of activity

\begin{tabular}{|c|c|c|c|c|c|c|}
\hline Comet & $\sigma$ & N. obs. & Class & $q(\mathrm{AU})$ & $\delta q(\mathrm{AU})$ & N. rev. \\
\hline 49P/Arend-Rigaux & 0.36 & 13 & $\mathrm{~L}$ & 1.37 & 0 & - \\
\hline 47P/Ashbrook-Jackson & 1.87 & 51 & $\mathrm{H}$ & 2.28 & -1.5 & 7 \\
\hline 19P/Borrelly & 0.47 & 12 & I & 1.32 & 0 & - \\
\hline 87P/Bus & 0.64 & 16 & I & 2.18 & -2.3 & 7 \\
\hline 101P/Chernykh & 1.18 & 26 & $\mathrm{H}$ & 2.36 & -0.2 & 1 \\
\hline 67P/Churyumov-Gerasimenko & 0.58 & 13 & I & 1.29 & -1.4 & 6 \\
\hline 32P/Comas-Sola & 1.13 & 46 & $\mathrm{H}$ & 1.77 & -0.4 & 10 \\
\hline $2 \mathrm{P} /$ Encke & 0.74 & 61 & I & 0.33 & 0 & - \\
\hline $4 \mathrm{P}^{\prime} /$ Faye & 0.70 & 33 & I & 1.59 & 0 & - \\
\hline 90P/Gehrels 1 & 1.92 & 12 & $\mathrm{H}$ & 2.94 & 0 & - \\
\hline 78P/Gehrels 2 & 0.43 & 10 & $\mathrm{~L}$ & 2.00 & -0.3 & 4 \\
\hline 82P/Gehrels 3 & 1.67 & 42 & $\mathrm{H}$ & 3.42 & -2.3 & 3 \\
\hline P/1997C1 (Gehrels) & 1.20 & 81 & $\mathrm{H}$ & 3.57 & -1.0 & 1 \\
\hline 21P/Giacobini-Zinner & 0.67 & 37 & I & 0.93 & -0.3 & 15 \\
\hline 65P/Gunn & 1.31 & 142 & $\mathrm{H}$ & 2.44 & -1.1 & 7 \\
\hline 110P/Hartley 3 & 1.63 & 16 & $\mathrm{H}$ & 2.45 & -0.2 & 2 \\
\hline P/1993K2 (Helin-Lawrence) & 0.91 & 45 & I & 3.09 & -0.6 & 1 \\
\hline 117P/Helin-Roman-Alu 1 & 1.11 & 81 & $\mathrm{H}$ & 3.71 & -0.8 & 11 \\
\hline 111P/Helin-Roman-Crockett & 2.72 & 60 & V & 3.47 & -1.8 & 3 \\
\hline 88P/Howell & 2.14 & 11 & $\mathrm{~V}$ & 1.41 & -0.3 & 3 \\
\hline P/1995A1 (Jedicke) & 1.04 & 42 & $\mathrm{H}$ & 4.08 & 0 & - \\
\hline P/1996A1 (Jedicke) & 0.95 & 140 & I & 4.06 & 0 & - \\
\hline 48P/Johnson & 0.26 & 26 & $\mathrm{~L}$ & 2.20 & 0 & - \\
\hline 59P/Kearns-Kwee & 1.39 & 10 & $\mathrm{H}$ & 2.21 & -2.1 & 4 \\
\hline $68 \mathrm{P} /$ Klemola & 0.34 & 15 & $\mathrm{~L}$ & 1.76 & 0 & - \\
\hline 70P/Kojima & 0.80 & 10 & I & 1.63 & +0.4 & 4 \\
\hline $22 \mathrm{P} /$ Kopff & 1.67 & 34 & $\mathrm{H}$ & 1.50 & -0.9 & 18 \\
\hline 99P/Kowal 1 & 1.31 & 21 & $\mathrm{H}$ & 4.66 & +0.3 & 3 \\
\hline 134P/Kowal-Vávrová & 1.02 & 30 & $\mathrm{H}$ & 2.61 & 0 & - \\
\hline P/1993X1 (Kushida-Muramatsu) & 1.03 & 17 & $\mathrm{H}$ & 2.75 & -1.6 & 5 \\
\hline P/1997T3 (Lagerkvist-Carsenty) & 0.54 & 46 & I & 4.24 & -5.1 & 3 \\
\hline P/1997V1 (Larsen) & 0.77 & 113 & I & 3.29 & 0 & - \\
\hline 77P/Longmore & 1.21 & 13 & $\mathrm{H}$ & 2.40 & -0.7 & 5 \\
\hline P/1997G1 (Montani) & 1.02 & 55 & $\mathrm{H}$ & 4.22 & 0 & 0 \\
\hline 120P/Mueller 1 & 1.00 & 17 & I & 2.74 & -0.9 & 5 \\
\hline 136P/Mueller 3 & 1.10 & 21 & $\mathrm{H}$ & 3.00 & -0.5 & 3 \\
\hline P/1993W1 (Mueller 5) & 1.75 & 44 & $\mathrm{H}$ & 4.25 & 0 & - \\
\hline $28 \mathrm{P} /$ Neujmin 1 & 0.25 & 12 & $\mathrm{~L}$ & 1.53 & 0 & - \\
\hline 39P/Oterma & 1.11 & 115 & $\mathrm{H}$ & 3.39 & -2.4 & 3 \\
\hline 119P/Parker-Hartley & 1.06 & 109 & $\mathrm{H}$ & 3.03 & -1.4 & 2 \\
\hline 7P/ Pons-Winnecke & 0.67 & 13 & I & 0.77 & 0 & - \\
\hline 30P/Reinmuth 1 & 0.87 & 15 & I & 1.86 & 0 & - \\
\hline 44P/Reinmuth 2 & 1.53 & 17 & $\mathrm{H}$ & 1.87 & 0 & - \\
\hline 94P/Russell 4 & 0.43 & 21 & $\mathrm{~L}$ & 2.13 & -0.3 & 4 \\
\hline 29P/Schwassmann-Wachmann 1 & 1.33 & 245 & $\mathrm{H}$ & 5.45 & 0 & 0 \\
\hline 31P/Schwassmann-Wachmann 2 & 1.34 & 48 & $\mathrm{H}$ & 2.07 & -1.4 & 11 \\
\hline P/1994J3 (Shoemaker 4) & 0.80 & 50 & I & 2.94 & 0 & - \\
\hline 128P/Shoemaker-Holt 1 & 2.59 & 42 & V & 3.05 & -1.1 & 2 \\
\hline 121P/Shoemaker-Holt 2 & 2.37 & 22 & $\mathrm{~V}$ & 2.65 & -0.6 & 2 \\
\hline 137P/Shoemaker-Levy 2 & 0.43 & 10 & $\mathrm{~L}$ & 1.84 & 0 & - \\
\hline 129P/Shoemaker-Levy 3 & 0.84 & 14 & I & 2.81 & -6.3 & 13 \\
\hline 118P/Shoemaker-Levy 4 & 1.56 & 33 & $\mathrm{H}$ & 2.02 & 0 & - \\
\hline 135P/Shoemaker-Levy 8 & 1.40 & 18 & $\mathrm{H}$ & 2.71 & -2.5 & 1 \\
\hline 74P/Smirnova-Chernykh & 1.18 & 109 & $\mathrm{H}$ & 3.55 & -1.7 & 4 \\
\hline 9P/Tempel 1 & 0.52 & 40 & I & 1.49 & -0.4 & 10 \\
\hline 10P/Tempel 2 & 0.58 & 63 & I & 1.31 & 0 & - \\
\hline $53 \mathrm{P} /$ van Biesbroeck & 1.28 & 52 & $\mathrm{H}$ & 2.40 & 0 & - \\
\hline 36P/Whipple & 1.04 & 56 & $\mathrm{H}$ & 2.45 & -2.4 & 10 \\
\hline 81P/Wild 2 & 1.60 & 27 & $\mathrm{H}$ & 1.49 & -3.5 & 4 \\
\hline 116P/Wild 4 & 2.06 & 19 & V & 1.99 & -1.4 & 2 \\
\hline 43P/Wolf-Harrington & 0.61 & 15 & I & 1.58 & -1.0 & 10 \\
\hline
\end{tabular}

$\sigma:$ Standard deviation of the all the observations at $r>3 \mathrm{AU}$

N. obs.: Number of data points.

Class: L - Low-active; I - Intermediate-active; H - High-active; and V - Vey-High-active. $\delta q$ : Change in perihelion distance. Negatives values correspond to downward jumps.

N. rev.: Number of revolutions from the last change in $q$ to the last perihelion passage. 


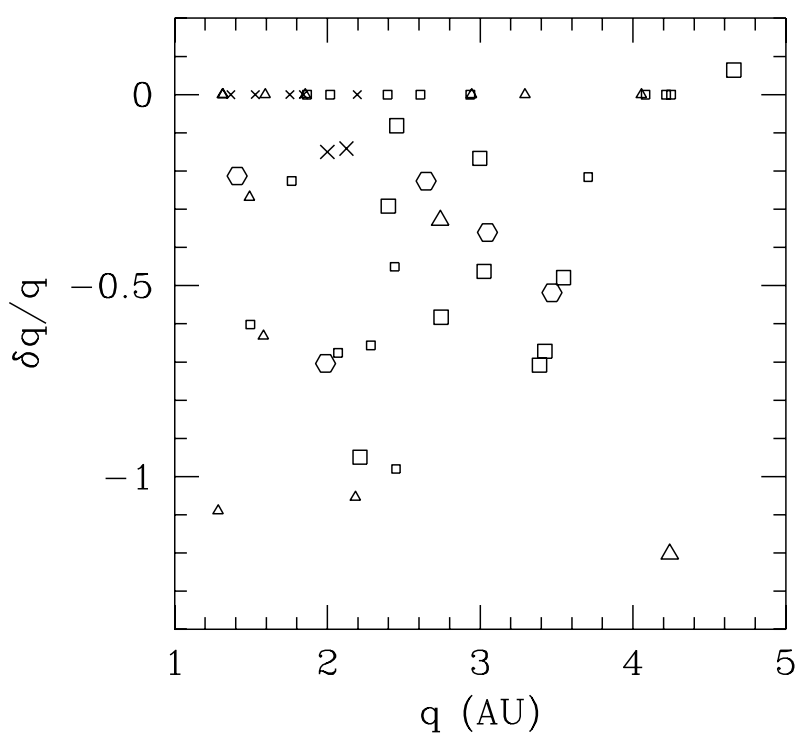

Fig. 8. The relative change in $q$ as a function of the minimum $q$ during the observed period. The different symbols correspond to: Low-active (L) comets whose changes in $q$ took place in the last five revolutions (large crosses), or between 6-20 revolutions ago or without important changes $(\delta q \sim 0)$ (small crosses); Intermediate-active (I) comets (similarly, large and small triangles); High-active (H) comets (large and small squares); and Very high-active (V) comets (large and small hexagons)

The increase in both quantity and quality of the nuclear magnitude data during the last fifteen years is spectacular, thanks mainly to the introduction of CCD cameras and the implementation of dedicated programs with medium-sized and large telescopes. Almost 2/3 of the data that we present was taken during the 1990's.

We plotted the compiled absolute nuclear magnitudes of $138 \mathrm{JF}$ comets as a function of the heliocentric distances at which they were determined. From these plots we were able to provide our "best estimates" for the absolute nuclear magnitudes of $105 \mathrm{JF}$ comets. There are in addition other $27 \mathrm{JF}$ comets - most of them lost - without estimated nuclear magnitudes. About $60 \%$ of the JF comets of our sample show very high or high activity at large heliocentric distances $(r>3 \mathrm{AU})$. This activity is correlated with downward jumps in the perihelion distance.

We find good consistency between Scotti's data (which partly relies on a method of coma substraction) and the rest of the CCD data. Roemer's nuclear magnitudes are on average about two magnitudes brighter than the ones obtained from CCD observations (including Scotti's) and our best estimates. Our adopted values for the nuclear magnitudes of those JF comets observed by the HST show good agreement with the corresponding estimates by the HST observers.

Our aim was to put together different sources of data on nuclear magnitudes of JF comets analysing their consistency and reliability. It is obvious that this effort is a first step, and great improvements in the statistics should be expected in the near future, as more data from HST, space missions, and ground-based observations with the new-generation very large telescopes will be obtained.

The catalog can be accessed online at: http://www.fisica.edu.uy/ gonzalo/catalog/. The plots for each comet can be accessed separately. We plan to include the raw and reduced data as plain text. Further updates of this catalog will be presented in the Web page.

Acknowledgements. The authors want to thank James Scotti and Lars Kamél for fruitful discussions on comet photometry, and Rudolph Dvorak for his hospitality during our stay at the Vienna Observatory during which part of this paper was carried out. Financial support from the Program for the Development of Basic Science (PEDECIBA) and the National Research Council of Uruguay (CONICYT) is gratefully acknowledged.

\section{Appendix A: The problem of coma extinction}

Let us consider a dust production rate $Q_{\mathrm{d}}$ (in number of particles $\mathrm{s}^{-1}$ ). The dust particles leave the gravitational influence zone of the comet with a terminal velocity $v_{\mathrm{d}}$. Let us assume that the optically important dust particles have an average radius $a \sim 0.5 \mu \mathrm{m}$ (Hanner et al. 1985) and a mass density $\rho_{\mathrm{d}} \sim 10^{3} \mathrm{~kg} \mathrm{~m}^{-3}$. For a spherically symmetrical dust coma with a uniform radial outflow, the number density of grains at a distance $x$ from the nucleus is

$n=\frac{Q_{\mathrm{d}}}{4 \pi x^{2} v_{\mathrm{d}}}$.

The extinction coefficient is then

$\tau=n \pi a^{2}=\frac{Q_{\mathrm{d}} a^{2}}{4 x^{2} v_{\mathrm{d}}}$

and the equation of radiative transfer is

$\frac{\mathrm{d} I}{I}=-\frac{Q_{\mathrm{d}} a^{2}}{4 v_{\mathrm{d}} x^{2}} \mathrm{~d} x$.

At the nucleus surface $\left(x=R_{\mathrm{N}}\right)$, the intensity is $I_{\mathrm{O}}$. Integrating Eq. (6) through the whole coma to $x \rightarrow \infty$, we get

$\frac{I}{I_{\mathrm{o}}}=\exp \left(-\frac{Q_{\mathrm{d}} a^{2}}{4 v_{\mathrm{d}} R_{\mathrm{N}}}\right)$

and the resulting extinction in magnitudes is

$\Delta m=\frac{2.5 \log e Q_{\mathrm{d}} a^{2}}{4 v_{\mathrm{d}} R_{\mathrm{N}}} \simeq 0.27 \frac{Q_{\mathrm{d}} a^{2}}{v_{\mathrm{d}} R_{\mathrm{N}}}$.

The mass of each individual grain is $m_{\mathrm{d}}=\frac{4}{3} \pi a^{3} \rho_{\mathrm{d}}$. The dust production rate in mass $Q_{\mathrm{d}}^{\prime}$ is then

$Q_{\mathrm{d}}^{\prime}=\frac{4}{3} \pi a^{3} \rho_{\mathrm{d}} Q_{\mathrm{d}}$

The gas $Q_{\mathrm{g}}$ and dust production rates are related through the dust to gas ratio $\alpha$ by $Q_{\mathrm{d}}^{\prime}=\alpha Q_{\mathrm{g}}$.

Assuming that the main gas component is water, the gas production rate can be computed as:

$Q_{\mathrm{g}}=4 \pi R_{\mathrm{N}}^{2} f Z_{\mathrm{w}} m_{\mathrm{w}}$ 
where $f$ is the fraction of active area, $Z_{\mathrm{w}}$ is the gas production rate per unit area and $m_{\mathrm{w}}$ is the mass of the water molecule. $Q_{\mathrm{d}}$ is then

$Q_{\mathrm{d}}=\frac{3 \alpha R_{\mathrm{N}}^{2} f Z_{\mathrm{w}} m_{\mathrm{w}}}{a^{3} \rho_{\mathrm{d}}}$

The dust outflow velocity is given by $v_{\mathrm{d}}=$ $450\left[Z_{\mathrm{w}} / Z_{w 0} \quad R_{\mathrm{N}}(\mathrm{km})\right]^{1 / 2} \mathrm{~m} \mathrm{~s}^{-1}$ (Hanner et al. 1985; Hanner 1985; Fernández et al. 1999), where $Z_{\mathrm{w} 0}$ is the gas production rate per unit area at $1 \mathrm{AU}$. Introducing the expression for $v_{\mathrm{d}}$ and Eq. (12) into Eq. (9), we get an extinction

$\Delta m \simeq \frac{1.8 m_{\mathrm{w}}}{Z_{\mathrm{w} 0}{ }^{\frac{1}{2}}} \frac{\alpha f Z_{\mathrm{w}}^{\frac{3}{2}}}{a \rho_{\mathrm{d}}}\left[R_{\mathrm{N}}(\mathrm{km})\right]^{\frac{1}{2}}$.

As an example, let us consider an active Jupiter family comet near perihelion (i.e., $q \sim 1.5 \mathrm{AU}$ ). The gas production rate per unit area at $1 \mathrm{AU}$ is $\sim 3.210^{21} \mathrm{~mol} \mathrm{~m}^{-2} \mathrm{~s}^{-1}$ and at $1.5 \mathrm{AU}$ is $\sim 1.210^{21} \mathrm{~mol} \mathrm{~m} \mathrm{~m}^{-2} \mathrm{~s}^{-1}$. If the comet has a fraction of active area of $\sim 10 \%$ and assuming a dust to gas ratio $\alpha=0.5$, we get

$\Delta m \simeq 0.004\left[R_{\mathrm{N}}(\mathrm{km})\right]^{\frac{1}{2}}$.

For a typical nuclear radius of $R_{\mathrm{N}} \sim 1 \mathrm{~km}$, the coma extinction should not exceed $10^{-2} \mathrm{mag}$, thus too low to affect the estimated magnitude of the nucleus, considering the other sources of much larger errors involved in this determination.

One of the weak assumptions of the previous model is the consideration of a constant outflow velocity $v_{\mathrm{d}}$ right from the surface. Considering a simple model where the velocity increases linearly from one tenth of its final value at the nucleus surface to the final value at a distance of 100 radii, we get an extinction ten times larger. On this extreme hypothesis, we only get significant extinction for very large and/or very active comets.

\section{References}

A'Hearn M.F., Millis R.L., 1980, AJ 85, 1528

Bailey M.E., 1986, Nat 324, 350

Chen J., Jewitt D., 1994, Icarus 108, 265

Delsemme A.H., Rud D.A., 1973, A\&A 28, 1

Eritsyan M.A., Akheverdyan L.G., 1987, Kinematika i Fisika Neb. Tel 3, 89

Festou M.C., Feldman P.D., 1987, in Exploring the Universe with the IUE Satellite, Kondo Y. (ed.). Reidel, Dordrecht, p. 101

Fernández J.A., Rickman H., Kamél L., 1992, in Periodic Comets, Fernández J.A., Rickman H. (eds.). Universidad de la República, Montevideo, Uruguay, p. 143

Fernández J.A., Tancredi G., Rickman H., Licandro J., 1999, A\&A 352,327

Fernández Y., Wellnitz D., Buie M., et al., 1999, Icarus 140, 205

Green D., 1988, International Comet Quartely Archive of Cometary Photometric Data, International Comet Quarterly, Cambridge, U.S.A.
Hanner M.S., 1985, Adv. Space Res. 4, 189

Hanner M.S., Tedesco E., Tokunaga A.T., et al., 1985, Icarus 64,11

Hartmann W.K., Tholen D.J., Cruikshank D.P., 1987, Icarus 69,33

Jewitt D., 1990, AJ 351, 277

Jewitt D., 1996, Earth, Moon and Planets 72, 185

Jewitt D., Danielson G.E., 1984, Icarus 60, 435

Jewitt D., Meech K., 1987, AJ 93, 1542

Jewitt D., Meech K.J., 1988, ApJ 328, 974

Jewitt D., Luu J., 1989, AJ 97, 1766

Kamél L., 1991, The comet light curve catalogue/atlas. I. The compiled observations. Uppsala Univ. Reprocentralen HSC

Keller H.U., Delamere W.A., Reitsema H.J., Huebner W.F., Schmidt H.U., 1987, A\&A 187, 807

Lamy P.L., Toth I., 1995, A\&A 293, L43

Lamy P.L., Toth I., Grün E., Keller H.U., Sekanina Z., West R.M., 1996, Icarus 119, 370

Lamy P.L., Toth I., A'Hearn M.F., Weaver H.A., 1997, in DPS meeting \# 29, p. 2902

Lamy P.L., Toth I., Jorda L., Weaver H.A., A'Hearn M., 1998a, A\&A 335, L25

Lamy P.L., Toth I., Weaver H., 1998b, A\&A 337, 945

Larson S.M., A'Hearn M.F., 1984, Icarus 58, 446

Licandro J., Tancredi G., Lindgren M., Rickman H., Gil-Hutton R., 1999a, Icarus 147, 161

Licandro J., Serra-Ricart M., Oscoz A., Duffard R., 1999b, poster presented at Asteroids, Comets, Meteors 1999

Luu J.X., Jewitt D.C., 1992, AJ 10, 2243

Marsden B.G., Williams G.V., 1997, Catalogue of Cometary Orbits, 12th Edition, Minor Planet Center, IAU

Meech K., Belton M.S., Mueller B., Dicksion M., Heide R., 1993, AJ 106, 1222

Mueller B., 1992, in Asteroids, Comets and Meteors 1991, Harris A.W., Bowell E. (eds.), p. 425

Mueller B., Ferrin I., 1996, Icarus 123, 463

Ninkov Z., 1994, AJ 107, 1182

Rabe E., 1972, in The Motion, Evolution of Orbits, and Origin of Comets, Chebotarev G.A., Kazimirchak-Polonskaya E.I., Marsden B.G. (eds.) IAU Symp. 45. Kluwer, The Netherlands, p. 55

Rickman H., Fernández J.A., Gustafson B.Å.S., 1990, A\&A 237,524

Roemer E., 1976, in The Study of Comets, Donn B., Mumma M., Jackson W., A'Hearn M., Harrington R. (eds.), NASA SP-393, US Government Printing Office, Washington DC, p. 380

Sekanina Z., 1976, in The Study of Comets, Donn B., Mumma M., Jackson W., A'Hearn M., Harrington R. (eds.), NASA SP-393, US Government Printing Office, Washington DC, p. 537

Sekanina Z., 1999, A\&A 342, 285

Shoemaker E.M., Wolfe R.F., 1982, in Satellites of Jupiter, Morrison D. (ed.). Univ. Arizona Press, Tucson, p. 277

Tancredi G., Rickman H., 1992, IAU Symp. 152, Ferraz-Mello S. (ed.). Kluwer Acad. Publ., p. 269

Weissman P., A'Hearn M., Rickman H., McFadden L.A., 1989, in Asteroids II, Binzel R., Gehrels T. Matthews M. (eds.). University of Arizona Press, p. 880

Valsecchi G.B., 1992, in Workshop on Periodic Comets, Fernández J.A., Rickman H. (eds.). Univ. República, Montevideo, p. 98 